\title{
Parameter Optimization for an Underwater Optical Wireless Vertical Link Subject to Link Misalignments
}

\author{
Ikenna Chinazaekpere Ijeh ${ }^{1}$, Mohammad Ali Khalighi ${ }^{1}$, Steve Hranilovic ${ }^{2}$ \\ ${ }^{1}$ Aix Marseille University, CNRS, Centrale Marseille, Institut Fresnel, Marseille, France \\ ${ }^{2}$ McMaster University, Dept. Electrical \& Computer Engineering, Hamilton, ON, Canada
}

\begin{abstract}
We consider transmitter-receiver ( $\mathbf{T x}-\mathbf{R x})$ parameter optimization for a vertical underwater wireless optical communication link under misalignment conditions. The $T x$ is assumed to use a light-emitting diode while the Rx uses a silicon photomultiplier that allows a high Rx sensitivity. We consider two typical application scenarios and investigate optimization of the Tx and the Rx parameters to optimize the link performance in terms of outage probability. We derive accurate analytical expressions for the distribution of the channel attenuation accounting for link misalignments, which allows for the evaluation of the link performance in terms of bit-error-rate and outage probability without resorting to costly Monte Carlo simulations. Through numerical results, we show and discuss the impact of parameter optimization on the link performance in different link configurations and for various misalignment conditions. In particular, the presented results show the significant performance improvement resulting from optimizing the $T x$ beam divergence and the $\mathrm{Rx} F \mathrm{FV}$ in the presence of pointing errors.
\end{abstract}

Index Terms-Underwater wireless optical communications; Link misalignment; Parameter optimization; Silicon photomultiplier; Vertical underwater links.

\section{INTRODUCTION}

In underwater environments, there is an essential need for efficient wireless communication techniques in a number of applications, including installation monitoring, underwater robotics, port security, etc. Underwater wireless optical communications (UWOC) offer low implementation cost, low latency, high data rate, and energy efficiency, compared with the traditional acoustic communications [1]-[5]. Extensive research has been realized in the past few years in order to address the main challenges of the UWOC technology [6]. Among these, one important issue is to deal with link misalignments, which are particularly problematic in underwater applications where precise localization and tracking of mobile units is very challenging [6]. Other issues include accurate modeling of signal degradation due to water absorption and scattering [7]-[11], solar background noise [12], and channel turbulence [13]-[17], as well as developing efficient transmission techniques to ascertain the reliability of the communication link [18], [19]. In particular, to increase the transmission range of UWOC links, the use of high sensitivity detectors including photo-multiplier tubes (PMTs), singlephoton avalanche diodes (SPADs), and arrays of SPADs, also known as silicon photo-multipliers (SiPMs) or multi-pixel photon counters (MPPCs), has received particular attention [20].

In this paper, we focus on the effect of Tx-Rx misalignment errors and propose optimization of the link parameters to minimize the link performance degradation. Indeed, although the impact of beam misalignment and pointing errors is a wellexplored topic in free-space optical (FSO) communications [21], [22], the past literature on UWOC systems mostly considered perfectly aligned line-of-sight (LoS) horizontal or vertical links. However, misalignment is, of course, one of major issues in underwater wireless optical links, which can considerably impact the link reliability. Misalignments can be due to different phenomena in practical link deployment scenarios. For instance, for the case of communication with a underwater mobile unit, they can arise from its positioning inaccuracy or instability. Also, in the case of transmission from a fixed node, e.g., at the sea surface, they can be due to node movements as a results of surface waves.

A few works have considered misalignment considerations for UWOC links. For instance, [23] presented a theoretical and experimental study of the effect of displacement in the Rx position and the inclination angle with respect to the main optical axis on the detected signal. The effect of beam divergence angle on the received power was investigated in [24], taking into consideration the Tx-Rx lateral offset. Also, for the case of turbid waters, [25] studied the effect of Rx angular misalignments and its field-of-view (FoV) on the received signal. Furthermore, for some special link configurations, e.g., a bottom-surface-bottom link in [26] or a surface-to-bottom link in [27], [28], the link performance was evaluated taking into account the effect of random sea surface, based on the verified experimental model of [29]. More recently, [30], [31] proposed an angular multiple-input multiple-output (MIMO) technique for relatively short UWOC links to relax the beam alignment requirements.

Our aim in this work is to focus on the system design for UWOC links corrupted by misalignments in order to minimize their impact on the link performance. As we will explain later, the main parameters that we consider here are the Tx beam divergence and the Rx FoV. Note that a few works have considered a similar approach in the contexts of outdoor FSO systems and indoor optical wireless systems. For instance, for a Gaussian beam propagating through an FSO channel subject 
to atmospheric turbulence and pointing errors, [32] proposed joint optimization of beam width and spatial coherence length of the Tx to maximize the average channel capacity. For FSO links deployed in high-altitude platforms subject to angle-ofarrival (AoA) fluctuations and pointing errors, [33] proposed an adaptive beam optimization technique at the $\mathrm{Tx}$ and at the Rx using a variable focus lens. Also, [34] considered optimization of the Tx Gaussian beam-waist and the Rx FoV for unmanned aerial vehicle (UAV)-based FSO links under channel turbulence and pointing error conditions. On the other hand, optimized Tx beam width was proposed in [35] for an indoor cellular optical wireless communication (OWC) and positioning system, which allowed a higher channel bandwidth (BW) and a higher position accuracy.

Given the substantially different beam propagation (including scattering), channel characteristics, Tx-Rx types, and operational particularities between FSO, indoor OWC and UWOC systems, there is the need for a unique study into the optimization of UWOC link parameters, which constitutes our motivation behind this work.

We consider a typical scenario for an UWOC link, employing light-emitting diodes (LEDs) at the Tx and a highsensitivity photo-detector, i.e., an SiPM at the Rx. The interest of using LEDs is their relatively high output power and the flexibility of being arranged in arrays to further increase the transmit power, and hence, the link span [36], [37]. Also, the SiPM, which is an array of Geiger mode biased APDs (SPADs), offers the advantages of high sensitivity, low implementation complexity, low operating bias voltage, mechanical robustness, and magnetic fields insensitivity, as compared to the PMT counterparts [20], [37], [38].

More specifically, in our case study, communication takes place between a buoy at the water surface and an AUV beneath it, as illustrated in Fig. 1. The typical application is high-speed data transfer from a sea surface observation station to an AUV (download mode), or transmitting the collected data by an AUV to a surface station for further long-range through-the-air transmission (upload mode), e.g., for data muling. The position and orientation of the buoy in this scenario are subject to sea surface waves, while those of the AUV are subject to water currents and AUV instability in its dynamic positioning (i.e., static position, used when establishing a communication link with the buoy).

To the best of our knowledge, the study of UWOC link optimization for a limited $\mathrm{Rx}$ FoV with a high-sensitivity photo-detector (PD) has not been considered in the literature thus far. We provide an accurate and computationally efficient analytical framework for the evaluation of the link performance in terms of the bit-error-rate (BER) and the outage probability $\left(P_{\text {out }}\right)$. This is based on precise mathematical modeling of angular misalignments and displacements of the AUV, as well as the angular misalignments at the buoy resulting from the sea surface waves. We show that careful selection of the Tx beam divergence and the Rx FoV allows significant performance improvement and substantially improved link reliability. Obviously, the link reliability can be further improved using powerful channel coding and/or more energy-efficient modulation techniques [19], [39].

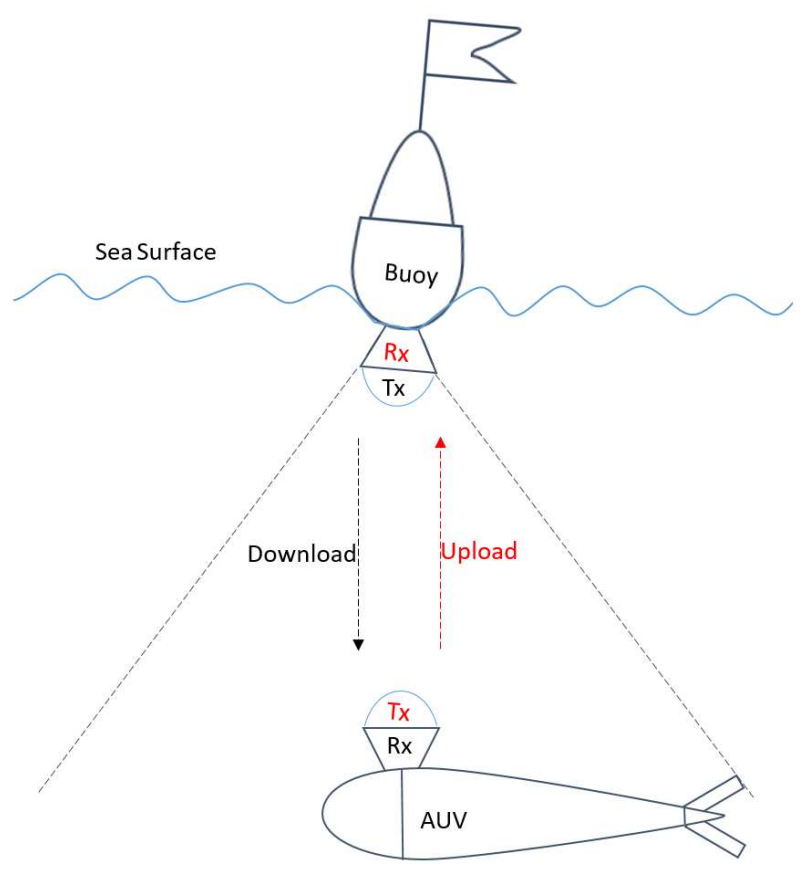

Fig. 1. Buoy-AUV illustration of the considered UWOC link.

The subsequent presentation of this paper is as follows. Section II introduces the considered UWOC link configuration and the related main assumptions. In Section III, we present mathematical modeling for pointing errors. Next, in Section $\mathrm{IV}$, the derivation of the distribution of channel coefficient is provided for the considered link configuration. Then, a set of numerical results are presented in Section $\mathrm{V}$ to show the performance improvement achieved by $\mathrm{Tx}-\mathrm{Rx}$ parameter optimization. Lastly, Section VI concludes the paper.

Notations: Boldface capital letters are used for matrices and boldface small letters for vectors. Also, $(.)^{\mathrm{T}}$ stands for transposition, $\|$.$\| is the Euclidean norm, \boldsymbol{a} \cdot \boldsymbol{b}$ denotes the dot product of vectors $\boldsymbol{a}$ and $\boldsymbol{b}$, and $\delta($.$) stands for the$ Dirac delta function. In addition, $\operatorname{Pr}($.$) denotes probability,$ and $\operatorname{erfc}(x)=\frac{2}{\sqrt{\pi}} \int_{x}^{\infty} e^{-t^{2}} d t$ and $Q(x)=\frac{1}{2} \operatorname{erfc}(x / \sqrt{2})$ are the well-known complementary error function and $Q$-function, respectively. Lastly, $\operatorname{sgn}(x)$ is the sign function, which equals 1 for $x \geq 0$ and -1 otherwise.

\section{Assumptions And Performance Metrics}

For the sake of brevity, we provide in the following the system model for the case of downlink transmission for the link configuration shown in Fig. 1, when the Tx is located at the buoy and the Rx at the AUV. The general block diagram of the communication link is shown in Fig. 2.

\section{A. Signal Transmission Modeling}

Figure 3 shows the corresponding link geometry, where the $\mathrm{Rx}$ is located at depth $D$, exactly beneath the Tx (i.e., when there is no displacement between the Tx and the Rx). The link distance $L$ is then equal to $D$. At the Tx, the LED is assumed to follow a Lambertian model with order $m$, which 
Information

bits

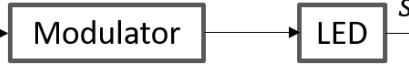

Transmitter

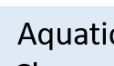

Channel

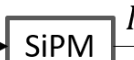

Fig. 2. The block diagram of the UWOC communication link.

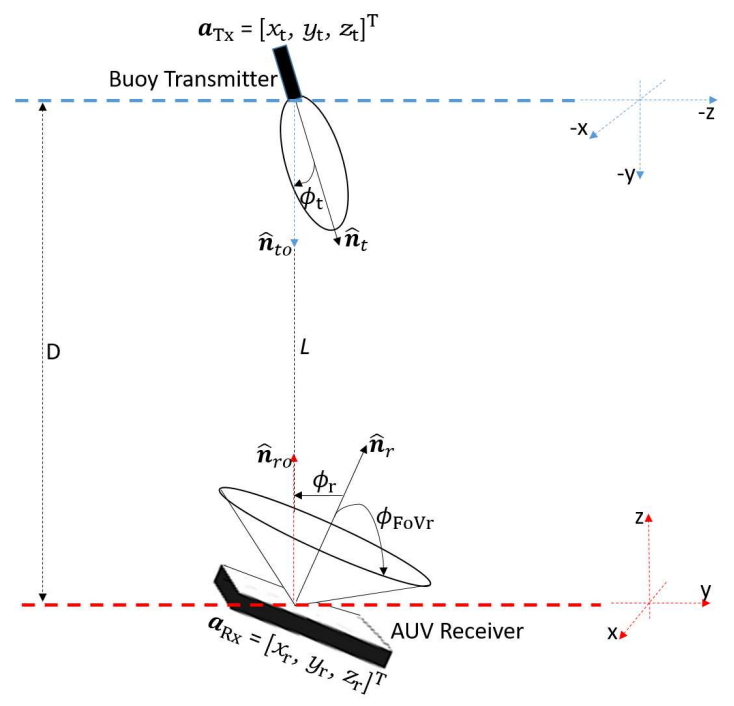

Fig. 3. Geometry of the buoy-AUV downlink UWOC system (inspired from [40]).

specifies the optical beam directionality [40]. At the $\mathrm{Rx}$, prior to the PD, we consider the use of an optical filter with signal transmission $T_{\mathrm{s}}\left(\phi_{\mathrm{r}}\right)$ in order to reject the background noise as far as possible [12], as well as a non-imaging concentrator of refractive index $n_{r}$ and gain $g\left(\phi_{\mathrm{r}}\right)$ with $\phi_{\mathrm{r}} \in\left\{0, \frac{\pi}{2}\right\}$ being the incidence angle [40]. Defining the active area of the PD by $A_{\mathrm{PD}}$, the effective $\mathrm{Rx}$ light collection area $A_{\mathrm{eff}}$ is then given by [40]:

$$
A_{\mathrm{eff}}\left(\phi_{\mathrm{r}}\right)=\left\{\begin{array}{cc}
A_{\mathrm{PD}} T_{\mathrm{s}}\left(\phi_{\mathrm{r}}\right) g\left(\phi_{\mathrm{r}}\right) \cos \left(\phi_{\mathrm{r}}\right), & \phi_{\mathrm{r}} \leq \phi_{\mathrm{FoVr}} \\
0, & \phi_{\mathrm{r}}>\phi_{\mathrm{FoVr}}
\end{array}\right.
$$

where

$$
g\left(\phi_{\mathrm{r}}\right)=\frac{n_{r}^{2}}{\sin ^{2}\left(\phi_{\mathrm{FoVr}}\right)},
$$

and $\phi_{\mathrm{FoVr}}$ is the Rx FoV. For a given transmit optical power $P_{\mathrm{Tx}}$, the received intensity $P_{\mathrm{Rx}}$ on the $\mathrm{PD}$ can then be expressed as [41]:

$$
P_{\mathrm{Rx}}=P_{\mathrm{Tx}} \frac{m+1}{2 \pi} \cos ^{m}\left(\phi_{\mathrm{t}}\right) e^{-\left(L K_{\mathrm{d}}\right)} \frac{A_{\mathrm{eff}}\left(\phi_{\mathrm{r}}\right)}{L^{2}} \Pi\left(\frac{\phi_{\mathrm{r}}}{\phi_{\mathrm{FoVr}}}\right)
$$

where, $\phi_{\mathrm{t}} \in\left\{\frac{-\pi}{2}, \frac{\pi}{2}\right\}$ is the angle of irradiance at the Tx. We have used the definition of $\Pi(u)=1$ if $u \leq 1$, and zero otherwise, which allows accounting for link interruption when the beam incident angle falls out of the Rx FoV. Also, $K_{\mathrm{d}}$ denotes the diffuse attenuation coefficient (taking into account both beam absorption and scattering in water) that depends on the beam wavelength and the concentration of the particulates in water (chlorophyll concentration for the so-called Case-1 waters [42]). The exponential term in (3) is an approximate model to the beam attenuation due to seawater absorption and scattering when using a relatively wide beam [43], which is typically the case when considering LEDs at the Tx [12].

Define the aggregate channel coefficient, which includes the effects of the Tx, the Rx, and the aquatic channel as:

$$
h=P_{\mathrm{Rx}} / P_{\mathrm{Tx}} .
$$

In general, $h$ is a random variable due to channel turbulence and Tx-Rx random misalignments.

As we use an LED at the Tx, we consider intensity modulation and direct detection for signal transmission. Without loss of generality, the non-return-to-zero (NRZ) on-off keying (OOK) is considered. At the Rx, the generated photo-current at the SiPM output is passed through a trans-impedance amplifier (TIA) followed by a low-pass filter (LPF) to limit the thermal noise variance (see Fig. 2).

The transmitted OOK signal $s_{i}, i \in\{0,1\}$, takes two values of $P_{\mathrm{Tx}_{0}}$ and $P_{\mathrm{Tx}_{1}}$, given the average transmitted optical power $P_{\mathrm{Tx}}$ and the extinction ratio, defined as $\xi=P_{\mathrm{Tx}_{0}} / P_{\mathrm{Tx}_{1}}$. The generated photo-current $I_{i}$ at the SiPM output is:

$$
I_{i}=\underbrace{\mathcal{R}_{\mathrm{e}} s_{i} h}_{I_{s, i}}+I_{d}+n_{s_{i}}=\breve{I}_{i}+n_{s_{i}},
$$

where $I_{s, i}$ represents the useful signal, $I_{d}$ is the dark current, and $n_{s_{i}}$ is the photo-current noise, including background noise, shot noise, and dark current noise. Also, $\breve{I}_{i}=I_{s, i}+I_{d}$, which includes the constant component of the dark current, and $\mathcal{R}_{\mathrm{e}}$ is the SiPM responsivity, given by [38]:

$$
\mathcal{R}_{\mathrm{e}}=\left(\frac{\Upsilon_{\mathrm{PDE}}}{E_{\mathrm{ph}}}\right)\left(1+P_{\mathrm{AP}}+P_{\mathrm{CT}}\right) e G .
$$

Here, $E_{\mathrm{ph}}$ is the photon energy and $e$ is the electron charge. Also, $G, \Upsilon_{\mathrm{PDE}}, P_{\mathrm{AP}}, P_{\mathrm{CT}}$ denote the SiPM gain, photon detection efficiency, probability of after-pulsing, and probability of cross-talk, respectively (see [20], [44] for detailed description of SiPM parameters). The SiPM dark current is given by:

$$
I_{d}=f_{\mathrm{DCR}}\left(1+P_{\mathrm{AP}}+P_{\mathrm{CT}}\right) e G,
$$

where $f_{\mathrm{DCR}}$ denotes the SiPM dark count rate.

Denote the signal (voltage) at the LPF output by $r_{i}$ :

$$
r_{i}=R_{\mathrm{L}} I_{i}+n_{\mathrm{th}}=\underbrace{R_{\mathrm{L}} \breve{I}_{i}}_{\breve{r}_{i}}+R_{\mathrm{L}} n_{s_{i}}+n_{\mathrm{th}}
$$


with $R_{\mathrm{L}}$ being the load resistance of the TIA, and $n_{\text {th }}$ denotes the thermal noise component in $r_{i}$, arising from the TIA and LPF circuitry (mainly related to $R_{\mathrm{L}}$ ). The corresponding total noise variance $\sigma_{n_{i}}^{2}$ (in units of $\mathrm{V}^{2}$ ) is given by:

$$
\sigma_{n_{i}}^{2}=R_{\mathrm{L}}^{2}\left(\sigma_{n_{s_{i}}}^{2}+\sigma_{\mathrm{th}}^{2}\right)=R_{\mathrm{L}}^{2}\left(\sigma_{\mathrm{sh}, i}^{2}+\sigma_{\mathrm{d}}^{2}+\sigma_{\mathrm{b}}^{2}\right)+\sigma_{\mathrm{th}}^{2},
$$

where $\sigma_{\mathrm{sh}, i}^{2}, \sigma_{\mathrm{d}}^{2}, \sigma_{\mathrm{b}}^{2}$, and $\sigma_{\mathrm{th}}^{2}\left(\right.$ in $\mathrm{V}^{2}$ ) denote the variances of the signal shot noise, dark noise, background noise, and thermal noise, respectively, where,

$$
\left\{\begin{aligned}
\sigma_{\mathrm{sh}, i}^{2} & =2 e G F B I_{s, i}, \\
\sigma_{\mathrm{d}}^{2} & =2 e G F B I_{\mathrm{d}}, \\
\sigma_{\mathrm{th}}^{2} & =4 K_{\mathrm{Z}} T B R_{\mathrm{L}} .
\end{aligned}\right.
$$

Here, $K_{\mathrm{Z}}, T, F$, and $B$ denote the Boltzmann constant, the Rx equivalent temperature in kelvin, the PD excess noise factor, and the $\mathrm{BW}$ of the Rx LPF, respectively. Let $B \approx R_{\mathrm{b}} / 2$, with $R_{b}$ being the bit rate [45].

In this work, channel delay dispersion is neglected, which is valid in practice when working in low-to-moderate turbidity waters [9], [11]. Also, we neglect the channel turbulence, which implies that sea currents and the gradient of salinity and temperature variations are considered as negligible. Furthermore, for simplicity, we will neglect the effect of background noise from solar radiations, i.e., we set $\sigma_{\mathrm{b}}^{2} \approx 0$. In fact, considering a worst-case scenario, with a relatively high level of radiation when the sun is at zenith and assuming no shadowing for a vertical downlink transmission, [46] showed that an SiPM-based Rx is highly impacted by the background noise in relatively shallow waters, despite using an narrowband optical filter. However, in our considered scenario these worst-case conditions are not likely to happen: the more critical case is the downlink (from the buoy-based Tx to the AUV Rx), where the buoy installation would shadow the sunlight, for example, when the sun is at the zenith. For the uplink case, the Rx performance could be limited by the laterally-diffused sunlight in water. However, the major part of this noise can be rejected by using a spatial filter at the buoy Rx.

\section{B. Performance Evaluation}

Given that channel variations due to misalignments occur in a much slower rate than the link bit-rate, the channel is modeled as non-ergodic for which the outage probability $P_{\text {out }}$ is an appropriate performance metric. Define $P_{\text {out }}$ as the probability that the link BER falls below a target BER. We further consider the probability of link interruption $P_{\text {int }}$ as the probability that the incident angle of the incoming beam at the Rx falls outside the Rx FoV, i.e., $\phi_{\mathrm{r}}>\phi_{\mathrm{FoVr}}$. Notice that interruption events are included in outage.

Recall that we consider NRZ-OOK modulation scheme. We do not consider any channel coding [19] in this work. We assume the Rx has perfect knowledge of the random channel attenuation coefficient $h$, based on which it sets the optimal detection threshold for signal demodulation. ${ }^{1}$ Note from the

\footnotetext{
${ }^{1}$ Note that this is a quite reasonable assumption since the channel varies slowly with respect to the bit duration, and can easily be estimated using pilo symbols [47].
}

previous subsection that, an important point is that the $\mathrm{Rx}$ noise is signal dependent, given the use of an SiPM PD. The instantaneous BER $P_{e}(e \mid h)$ can be written as [48]:

$$
P_{e}(e \mid h)=\frac{1}{4} \operatorname{erfc}\left(\frac{\gamma_{\mathrm{th}}-\breve{r}_{0}}{\sqrt{2 \sigma_{n_{0}}^{2}}}\right)+\frac{1}{4} \operatorname{erfc}\left(\frac{\breve{r}_{1}-\gamma_{\mathrm{th}}}{\sqrt{2 \sigma_{n_{1}}^{2}}}\right),
$$

where $\gamma_{\text {th }}$ stands for the optimal detection threshold, and $\breve{r}_{0}$ and $\breve{r}_{1}$ denote the signals input to the demodulator, corresponding to bits ' 0 ' and ' 1 ', respectively. The corresponding noise variances are denoted by $\sigma_{n_{0}}^{2}$ and $\sigma_{n_{1}}^{2}$, calculated from (9). The optimal threshold $\gamma_{\text {th }}$ is calculated as [48]:

$$
\begin{array}{r}
\gamma_{\text {th }}=\frac{\breve{r}_{0} \sigma_{n_{1}}^{2}-\breve{r}_{1} \sigma_{n_{0}}^{2}}{\sigma_{n_{1}}^{2}-\sigma_{n_{0}}^{2}}+\left[\frac{\breve{r}_{1}^{2} \sigma_{n_{0}}^{2}}{\sigma_{n_{1}}^{2}-\sigma_{n_{0}}^{2}}+\right. \\
\left(\frac{\breve{r}_{0} \sigma_{n_{1}}^{2}-\breve{r}_{1} \sigma_{n_{0}}^{2}}{\sigma_{n_{1}}^{2}-\sigma_{n_{0}}^{2}}\right)^{2}-\frac{\breve{r}_{0}^{2} \sigma_{n_{1}}^{2}}{\sigma_{n_{1}}^{2}-\sigma_{n_{0}}^{2}} \\
\left.\quad-\frac{\sigma_{n_{0}}^{2} \sigma_{n_{1}}^{2}}{\sigma_{n_{1}}^{2}-\sigma_{n_{0}}^{2}} \ln \left(\frac{\sigma_{n_{0}}^{2}}{\sigma_{n_{1}}^{2}}\right)\right]^{0.5} .
\end{array}
$$

The average $\mathrm{BER}^{2}$ can then be calculated as:

$$
P_{e}=\int_{0}^{\infty} P_{e}(e \mid h) f_{h}(h) d h,
$$

where $f_{h}(h)$ is the PDF of $h$. Also, the outage probability can be defined as:

$$
P_{\text {out }}=\operatorname{Pr}\left(h<h_{\mathrm{th}}\right)=\int_{0}^{h_{\mathrm{th}}} f_{h}(h) d h,
$$

where $h_{\mathrm{th}}$ is defined so that $\mathrm{BER}_{\mathrm{th}}=P_{e}\left(e \mid h_{\mathrm{th}}\right)$. Lastly, the link interruption probability is:

$$
P_{\text {int }}=\operatorname{Pr}\left(\phi_{\mathrm{r}}>\phi_{\mathrm{FoVr}}\right) .
$$

\section{Analytical Outage Probability Calculation}

As will be explained in Section V, the link performance can be evaluated through a statistical Monte Carlo (MC) approach. Obviously, there is interest to calculate these quantities analytically to avoid timely MC simulations. We provide here details on the derivation of the average BER and the outage probability. Whereas closed form expressions can readily be derived for the case of using a simple PIN PD [27], [34], the signal dependent noise in our case (resulting in a complex form for $P_{e}(e \mid h)$ and for the optimal detection threshold $\gamma_{\text {th }}$ in (11) and (12)) and the relatively complex expressions for the PDF of $h$ (as will be shown later in Section IV) make derivation of closed form expressions for $P_{\text {out }}$ very complex. For this reason, we propose here to make some simplifying assumptions. Firstly, we assume that the shot noise $R_{\mathrm{L}}^{2} \sigma_{\mathrm{sh}, i}^{2}$ in (8) is the dominant noise factor at the Rx. This implies that we have a large PD gain $G$ (which is actually the case), and that for OOK signaling, a relatively large $\mathrm{e}^{3}$ extinction ratio $\xi$ is used, which implies that even for "off" OOK symbols, the thermal and dark noises are negligible, compared with the

\footnotetext{
${ }^{2}$ Although our channel is non-ergodic and the appropriate metric is the outage probability, $P_{e}$ is also given for the sake of completeness.

${ }^{3}$ We have verified by numerical simulations that the obtained solution is quite accurate for $\xi \gtrsim 30 \%$, and even provides a good accuracy for $\xi$ as small as $\sim 10 \%$.
} 
corresponding signal-dependent shot noise. As a result, given that $I_{s, 0}=\xi I_{s, 1}$, we set $\breve{r}_{0} \approx \xi \breve{r}_{1}$ and also $\sigma_{n_{0}}^{2} \approx \xi \sigma_{n_{1}}^{2}$. Then, considering optimal thresholding for signal detection, the error probability in (11) can be simplified as:

$$
P_{e}(e \mid h)=\frac{1}{2} \operatorname{erfc}\left(\frac{R_{\mathrm{L}} I_{s, 1}-\gamma_{\mathrm{th}}}{\sqrt{2 \sigma_{n_{1}}^{2}}}\right)=Q\left(\frac{R_{\mathrm{L}} I_{s, 1}-\gamma_{\mathrm{th}}}{\sqrt{\sigma_{n_{1}}^{2}}}\right) .
$$

Also, with the considered approximations, the expression of $\gamma_{\text {th }}$ in (12) can be simplified as follows:

$$
\gamma_{\text {th }}=R_{\mathrm{L}} \sqrt{\xi I_{s, 1}^{2}-\frac{\xi \sigma_{n_{1}}^{2}}{1-\xi} \log \xi} \approx R_{\mathrm{L}} I_{s, 1} \sqrt{\xi}
$$

Now, to calculate $P_{\text {out }}$ for a given $h_{\text {th }}$ (which corresponds to a BER $\left.\mathrm{Bh}_{\mathrm{th}}\right)$, we have $\sigma_{n_{1}}^{2}=R_{\mathrm{L}}^{2}\left(2 e G F B \mathcal{R}_{\mathrm{e}} s_{1} h_{\mathrm{th}}+\sigma_{\mathrm{d}}^{2}\right)+\sigma_{\text {th }}^{2}$ and $I_{s, 1}=\mathcal{R}_{\mathrm{e}} s_{1} h_{\mathrm{th}}$. Then, from (16) we can define:

$$
\mathcal{A} \triangleq Q^{-1}\left(\mathrm{BER}_{\mathrm{th}}\right)=\frac{R_{\mathrm{L}} \mathcal{R}_{\mathrm{e}} s_{1} h_{\mathrm{th}}-R_{\mathrm{L}} \mathcal{R}_{\mathrm{e}} s_{1} h_{\mathrm{th}} \sqrt{\xi}}{\sqrt{R_{\mathrm{L}}^{2}\left(2 e G F B \mathcal{R}_{\mathrm{e}} s_{1} h_{\mathrm{th}}+\sigma_{\mathrm{d}}^{2}\right)+\sigma_{\mathrm{th}}^{2}}}
$$

$\mathcal{A}$ can be calculated, for instance, using the Matlab qfuncinv function [49]. We also define for notation simplicity $\mu=$ $R_{\mathrm{L}} \mathcal{R}_{\mathrm{e}} s_{1}$ and $\beta=2 e R_{\mathrm{L}} G B F \mu$. We can then rewrite (18) as:

$$
\mathcal{A}=\frac{\mu h_{\mathrm{th}}(1-\sqrt{\xi})}{\sqrt{\beta h_{\mathrm{th}}+R_{\mathrm{L}}^{2} \sigma_{\mathrm{d}}^{2}+\sigma_{\mathrm{th}}^{2}}}
$$

It can be easily shown that $h_{\text {th }}$ can be calculated from the following equation:

$$
h_{\mathrm{th}}=\frac{\mathcal{B}+\sqrt{\mathcal{B}^{2}+4 \mathcal{D C}}}{2 \mathcal{D}},
$$

where $\mathcal{B}=\mathcal{A}^{2} \beta, \mathcal{C}=\mathcal{A}^{2}\left(R_{\mathrm{L}}^{2} \sigma_{\mathrm{d}}^{2}+\sigma_{\text {th }}^{2}\right)$, and $\mathcal{D}=$ $\mu^{2}(1-\sqrt{\xi})^{2}$. The accuracy of this approach will later be quantified by numerical results in Section V.

\section{Pointing ERror Modeling}

Consider the downlink configuration as the default case, i.e., when the Tx is on the buoy and the Rx is on the AUV. For the mathematical derivations, we assume that the buoy's position is nearly fixed, whereas its orientation is primarily affected by the surface waves as a result of wind. The orientation of the $\mathrm{Rx}$ is deviated by underwater currents and the instability of the AUV, whereas its displacement is due to positioning inaccuracies. To account for pointing errors, we consider the perfect alignment case when the AUV is exactly beneath the buoy and there is no orientation deviation with respect to the optical axis. Figure 3 illustrates the scenario where there is no position displacement between the Tx and the Rx but there are orientation deviations at both the $\mathrm{Tx}$ and the $\mathrm{Rx}$ with respect to the optical axis. A more general scenario is illustrated in Fig. 4, where there is also a position displacement of the $\mathrm{Rx}$ with respect to the Tx.

Consider the more general misalignment case as illustrated in Fig. 4. Under perfect Tx-Rx alignment, denote the normal vectors to the Tx and the Rx by $\hat{\boldsymbol{n}}_{t o}$ and $\hat{\boldsymbol{n}}_{r o}$, respectively, where $\hat{\boldsymbol{n}}_{t o}=[0,0,-1]^{\mathrm{T}}$ and $\hat{\boldsymbol{n}}_{r o}=[0,0,1]^{\mathrm{T}}$. Under misaligned beam conditions, these normal vectors are denoted by

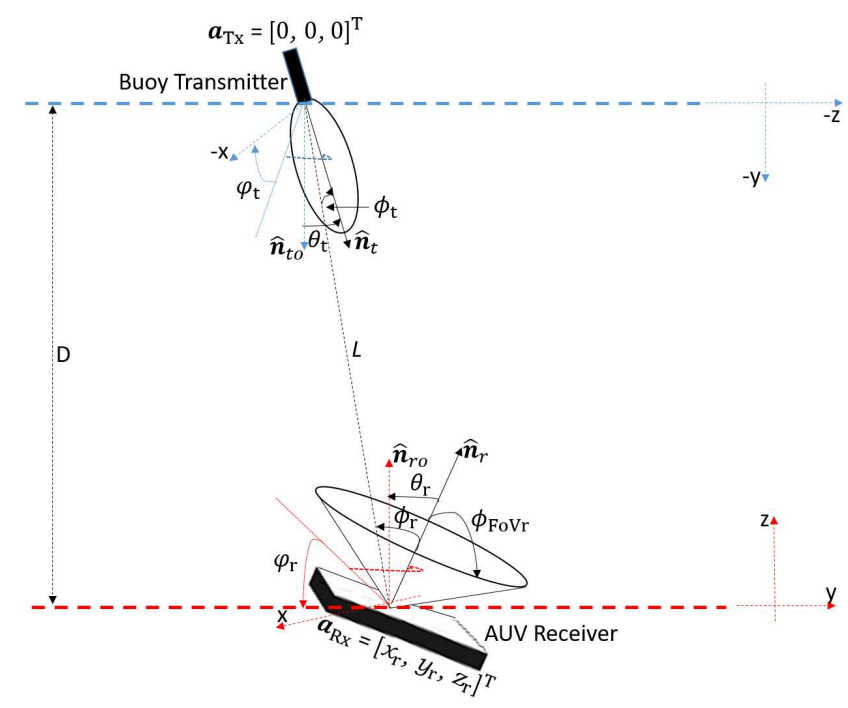

Fig. 4. Transmitter and Receiver with Orientation and Position Deviations (inspired from [40]).

$\hat{\boldsymbol{n}}_{t}$ and $\hat{\boldsymbol{n}}_{r}$, respectively. Let $\theta_{\mathrm{t}}$ denote the polar angle between $\hat{\boldsymbol{n}}_{t o}$ and $\hat{\boldsymbol{n}}_{t}$, and $\varphi_{\mathrm{t}}$ the azimuthal angle to $\hat{\boldsymbol{n}}_{t}$. Similarly, the polar and azimuthal angles of the Rx are denoted by $\theta_{\mathrm{r}}$ and $\varphi_{\mathrm{r}}$, respectively. Like in Fig. 3, here, $\phi_{\mathrm{t}}$ and $\phi_{\mathrm{r}}$ denote the polar angles with respect to the optical axis. We have:

$$
\begin{aligned}
& \cos \left(\phi_{\mathrm{t}}\right)=\frac{\hat{\boldsymbol{n}}_{t} \cdot\left(\boldsymbol{a}_{\mathrm{Rx}}-\boldsymbol{a}_{\mathrm{Tx}}\right)}{\left\|\boldsymbol{a}_{\mathrm{Rx}}-\boldsymbol{a}_{\mathrm{Tx}}\right\|}, \\
& \cos \left(\phi_{\mathrm{r}}\right)=\frac{\hat{\boldsymbol{n}}_{r} \cdot\left(\boldsymbol{a}_{\mathrm{Tx}}-\boldsymbol{a}_{\mathrm{Rx}}\right)}{\left\|\boldsymbol{a}_{\mathrm{Tx}}-\boldsymbol{a}_{\mathrm{Rx}}\right\|}
\end{aligned}
$$

where $\left\|\boldsymbol{a}_{\mathrm{Tx}}-\boldsymbol{a}_{\mathrm{Rx}}\right\|=L$. Denote the Cartesian coordinates of the Tx and the Rx by vectors $\boldsymbol{a}_{\mathrm{Tx}}=\left[x_{\mathrm{t}}, y_{\mathrm{t}}, z_{\mathrm{t}}\right]^{\mathrm{T}}$ and $\boldsymbol{a}_{\mathrm{Rx}}=$ $\left[x_{\mathrm{r}}, y_{\mathrm{r}}, z_{\mathrm{r}}\right]^{\mathrm{T}}$, respectively. Also, without loss of generality, in the following, consider the buoy's position as the origin of the Cartesian coordinates, i.e., $\boldsymbol{a}_{\mathrm{Tx}}=[0,0,0]^{\mathrm{T}}$. Note that when there is no Rx displacement, like in Fig. 3, we have $\phi_{\mathrm{t}}=\theta_{\mathrm{t}}$ and $\phi_{\mathrm{r}}=\theta_{\mathrm{r}}$.

\section{A. Modeling Tx Orientation}

The buoy at the sea surface swerves as wind blows across it from different directions. A set of experimental measurements were carried out in [29] to measure the sea surface slope. A close linearity was then deduced between the variance of sea surface slopes and the wind speed $U$ for an approximate range of $1 \mathrm{~m} / \mathrm{s}<U<14 \mathrm{~m} / \mathrm{s}$. This model was later validated in [50] based on a derived and experimentally-validated omni-directional wind-dependent sea surface spectrum. It was adopted in [27], [28] for a buoy-based vertical UWOC link, where the distribution of the random sea surface slope was expressed in terms of spherical coordinates as follows:

$$
f_{\theta_{\mathrm{t}}, \varphi_{\mathrm{t}}}\left(\theta_{\mathrm{t}}, \varphi_{\mathrm{t}}\right)=\frac{\tan \left(\theta_{\mathrm{t}}\right) \sec ^{2}\left(\theta_{\mathrm{t}}\right)}{2 \pi \sigma_{\mathrm{u}}^{2}} \exp \left(\frac{-\tan ^{2}\left(\theta_{\mathrm{t}}\right)}{2 \sigma_{\mathrm{u}}^{2}}\right),
$$

where the variance $\sigma_{\mathrm{u}}^{2}$ of the omni-directional sea surface slope (which is independent of the wind direction) is given by [29]:

$$
\sigma_{\mathrm{u}}^{2}=0.003+0.00512 U \pm 0.004,
$$


with $U$ being in units of $\mathrm{m} / \mathrm{s}$. Note that in (23), $\varphi_{\mathrm{t}}$ is assumed to be uniformly distributed between 0 and $2 \pi$.

\section{B. Modeling Rx Orientation and Displacement}

We model the orientation of the Tx and the $\mathrm{Rx}$ by a rotation matrix $\boldsymbol{R}$ applied to their initial vectors $\hat{\boldsymbol{n}}_{t o}$ and $\hat{\boldsymbol{n}}_{r o}$ (corresponding to the perfect link alignment, see Fig. 3), respectively [51]. This way, $\hat{\boldsymbol{n}}_{t}=\boldsymbol{R} \hat{\boldsymbol{n}}_{t o}$, and $\hat{\boldsymbol{n}}_{r}=\boldsymbol{R} \hat{\boldsymbol{n}}_{r o}$. Matrix $\boldsymbol{R}$ accounts for rotations around $z, x$, and $y$ axes (yaw $\gamma$, pitch $\alpha$, and roll $\beta$ ) through rotation matrices $\boldsymbol{R}_{\gamma}, \boldsymbol{R}_{\alpha}$, and $\boldsymbol{R}_{\beta}$, respectively:

$$
\begin{gathered}
\boldsymbol{R}_{\gamma}=\left[\begin{array}{ccc}
\cos \gamma & -\sin \gamma & 0 \\
\sin \gamma & \cos \gamma & 0 \\
0 & 0 & 1
\end{array}\right], \boldsymbol{R}_{\alpha}=\left[\begin{array}{ccc}
1 & 0 & 0 \\
0 & \cos \alpha & -\sin \alpha \\
0 & \sin \alpha & \cos \alpha
\end{array}\right], \\
\boldsymbol{R}_{\beta}=\left[\begin{array}{ccc}
\cos \beta & 0 & \sin \beta \\
0 & 1 & 0 \\
-\sin \beta & 0 & \cos \beta
\end{array}\right],
\end{gathered}
$$

here, $\alpha$ and $\beta$ represent the sea surface slope effect from the up/downwind and the crosswind directions [29].

Given $\hat{\boldsymbol{n}}_{t o}=[0,0,-1]^{\mathrm{T}}, \hat{\boldsymbol{n}}_{t}$ can be written as:

$$
\begin{aligned}
\hat{\boldsymbol{n}}_{t} & =\boldsymbol{R} \hat{\boldsymbol{n}}_{t o}=\boldsymbol{R}_{\gamma} \boldsymbol{R}_{\alpha} \boldsymbol{R}_{\beta} \hat{\boldsymbol{n}}_{t o} \\
& =\left[\begin{array}{c}
-\cos \beta \sin \gamma \sin \alpha-\cos \gamma \sin \beta \\
\cos \gamma \cos \beta \sin \alpha-\sin \gamma \sin \beta \\
-\cos \alpha \cos \beta
\end{array}\right]=\left[\begin{array}{c}
-\sin \theta_{\mathrm{t}} \cos \varphi_{\mathrm{t}} \\
-\sin \theta_{\mathrm{t}} \sin \varphi_{\mathrm{t}} \\
-\cos \theta_{\mathrm{t}}
\end{array}\right]
\end{aligned}
$$

Similarly,

$$
\begin{aligned}
\hat{\boldsymbol{n}}_{r} & =\boldsymbol{R} \hat{n}_{r o}=\boldsymbol{R}_{\gamma} \boldsymbol{R}_{\alpha} \boldsymbol{R}_{\beta} \hat{n}_{\boldsymbol{r}_{o}} \\
& =\left[\begin{array}{c}
\cos \beta \sin \gamma \sin \alpha+\cos \gamma \sin \beta \\
\sin \gamma \sin \beta-\cos \gamma \cos \beta \sin \alpha \\
\cos \alpha \cos \beta
\end{array}\right]=\left[\begin{array}{c}
\sin \theta_{\mathrm{r}} \cos \varphi_{\mathrm{r}} \\
\sin \theta_{\mathrm{r}} \sin \varphi_{\mathrm{r}} \\
\cos \theta_{\mathrm{r}}
\end{array}\right] .
\end{aligned}
$$

Based on the illustration of Fig. 4, and given that $z_{\mathrm{t}}=D,(21)$ and (22) can be expressed as:

$$
\cos \left(\phi_{\mathrm{t}}\right)=\underbrace{\left(-\frac{x_{\mathrm{r}}}{L} \cos \left(\varphi_{\mathrm{t}}\right)-\frac{y_{\mathrm{r}}}{L} \sin \left(\varphi_{\mathrm{t}}\right)\right)}_{a_{\mathrm{t}}} \sin \left(\theta_{\mathrm{t}}\right)+\underbrace{\frac{D}{L}}_{b} \cos \left(\theta_{\mathrm{t}}\right)
$$

$\cos \left(\phi_{\mathrm{r}}\right)=\underbrace{\left(-\frac{x_{\mathrm{r}}}{L} \cos \left(\varphi_{\mathrm{r}}\right)-\frac{y_{\mathrm{r}}}{L} \sin \left(\varphi_{\mathrm{r}}\right)\right)}_{a_{\mathrm{r}}} \sin \left(\theta_{\mathrm{r}}\right)+\underbrace{\frac{D}{L}}_{b} \cos \left(\theta_{\mathrm{r}}\right)$,

where we have defined the parameters $a_{\mathrm{t}}, a_{\mathrm{r}}$, and $b$ for notation simplicity, similar to the approach in [51]. We model $x_{\mathrm{r}}$ and $y_{\mathrm{r}}$ by zero-mean independent Gaussian random variables (RVs) with variance $\sigma_{\mathrm{pr}}^{2}$. Also, for simplicity, $\theta_{\mathrm{r}}$ is modeled as a zeromean Gaussian-distributed RV with variance $\sigma_{\theta \mathrm{r}}^{2}$.

\section{Channel Coefficient Distribution}

In order to analytically evaluate the link performance, we need the probability density function (PDF) of the received signal in the general case of a link subject to Tx-Rx angular misalignments and displacements. For the sake of simplicity, we assume that the concentrator gain $g\left(\phi_{\mathrm{r}}\right)$ and the optical filter transmittance $T_{\mathrm{s}}\left(\phi_{\mathrm{r}}\right)$ are independent of $\phi_{\mathrm{r}}$, and denote them simply by $T_{s}$ and $g$, respectively. Regarding the optical filter, this simplification could be equivalent to removing the optical filter, as we assume negligible background noise, see the last part of Subsection II-A. ${ }^{4}$ From (3) and (4), we have:

$$
h=h_{c} \cos ^{m}\left(\phi_{\mathrm{t}}\right) \cos \left(\phi_{\mathrm{r}}\right) \Pi\left(\frac{\phi_{\mathrm{r}}}{\phi_{\mathrm{FoVr}}}\right),
$$

where $h_{c}$ denotes the deterministic part of $h$ :

$$
h_{c}=\frac{m+1}{2 \pi} \frac{A_{\mathrm{PD}} T_{\mathrm{s}} g}{L^{2}} e^{-\left(L K_{\mathrm{d}}\right)} .
$$

Let us further separate in (29) the parts depending only on $\phi_{\mathrm{t}}$ and $\phi_{\mathrm{r}}$, that we denote by $h_{\phi_{\mathrm{t}}}$ and $h_{\phi_{\mathrm{r}}}$, respectively:

$$
\begin{gathered}
h=h_{c} h_{\phi_{\mathrm{t}}} h_{\phi_{\mathrm{r}}}, \\
h_{\phi_{\mathrm{t}}}=\cos ^{m}\left(\phi_{\mathrm{t}}\right), \\
h_{\phi_{\mathrm{r}}}=\cos \left(\phi_{\mathrm{r}}\right) \Pi\left(\frac{\phi_{\mathrm{r}}}{\phi_{\mathrm{FoVr}}}\right) .
\end{gathered}
$$

To derive the PDF of $h$, we consider the following cases in the subsequent subsections. First, in Subsection IV-A, only Tx angular misalignments are considered (without any displacement) while assuming a fixed Rx (orientation and position) and a (fixed) large Rx FoV. Then, in Subsection IV-B, both $\mathrm{Tx}$ and $\mathrm{Rx}$ angular misalignments are taken into account without any displacement, i.e., as illustrated in Fig. 3. The PDF is then modified in in Subsection IV-C to account for $\mathrm{Rx}$ displacements, i.e., as in Fig. 4. Lastly, the generalization to the case of limited Rx FoV is considered in Subsection IV-D.

\section{A. Tx Angular Misalignment and Fixed Rx}

The key point here is to relate the distribution of $h$ back to the random angular misalignment of the buoy. For this case, $\phi_{\mathrm{t}}=\theta_{\mathrm{t}}$ and $\phi_{\mathrm{r}}=\theta_{\mathrm{r}}=0$, thus $h_{\phi_{\mathrm{r}}}=1$, and $h=h_{\mathrm{c}} h_{\phi_{\mathrm{t}}}$ that we denote by $h_{0}$ whose cumulative density function (CDF) can be obtained as:

$$
\begin{aligned}
& F_{h_{0}}\left(h_{0}\right)=\operatorname{Pr}\left(h_{c} h_{\phi_{\mathrm{t}}} \leq h_{0}\right)=\operatorname{Pr}\left(h_{c} \cos ^{m}\left(\theta_{\mathrm{t}}\right) \leq h_{0}\right) \\
& =\operatorname{Pr}\left(\theta_{\mathrm{t}}>\cos ^{-1}\left(\sqrt[m]{\frac{h_{0}}{h_{c}}}\right)\right)=1-F_{\theta_{\mathrm{t}}}\left(\cos ^{-1}\left(\sqrt[m]{\frac{h_{0}}{h_{c}}}\right)\right),
\end{aligned}
$$

where $F_{\theta_{\mathrm{t}}}($.$) denotes the CDF of \theta_{\mathrm{t}}$. From (34), the PDF of $h_{0}$ can be obtained as follows:

\footnotetext{
${ }^{4}$ Note that, in general, the approximation of a constant $T_{s}$ is justified when the FoV is relatively small, with the range depending on the filter bandwidth. Nevertheless, recent techniques have shown the possibility of designing relatively wide FoV optical filters using metamaterials, e.g. [52]. Investigation of link parameter optimization while taking into account the dependence of $T_{\mathrm{s}}$ on $\phi_{\mathrm{r}}$, which is particularly interesting when background noise effect cannot be neglected (e.g., in downlink), can be subject of future research.
} 


$$
f_{h_{0}}\left(h_{0}\right)=\frac{C_{1}}{m h_{0} \sqrt{\left(\sqrt[m]{\frac{h_{0}}{h_{c}}}\right)^{-2}-1}} f_{\theta_{\mathrm{t}}}\left(\cos ^{-1}\left(\sqrt[m]{\frac{h_{0}}{h_{c}}}\right)\right)
$$

where $C_{1}$ is a normalization coefficient to have $F_{h_{0}}(\infty)=$ 1 , and $f_{\theta_{\mathrm{t}}}($.$) is the PDF of \theta_{\mathrm{t}}$ that is obtained from (23) as $f_{\theta_{\mathrm{t}}}\left(\theta_{\mathrm{t}}\right)=2 \pi f_{\theta_{\mathrm{t}}, \varphi_{\mathrm{t}}}\left(\theta_{\mathrm{t}}, \varphi_{\mathrm{t}}\right)$.

\section{B. Tx-Rx Angular Misalignments and Fixed Rx Position}

We consider now random $\phi_{\mathrm{t}}=\theta_{\mathrm{t}}$ and $\phi_{\mathrm{r}}=\theta_{\mathrm{r}}$, while considering $\phi_{\mathrm{FoVr}}>\phi_{\mathrm{r}}$. In this case, $h=h_{c} \cos ^{m}\left(\theta_{\mathrm{t}}\right) \cos \left(\theta_{\mathrm{r}}\right)=$ $h_{0} h_{\phi_{\mathrm{r}}}$. The CDF of $h_{\phi_{\mathrm{r}}}, F_{h_{\phi_{\mathrm{r}}}}($.$) , can be written as:$

$$
F_{h_{\phi_{\mathrm{r}}}}\left(h_{\phi_{\mathrm{r}}}\right)=\operatorname{Pr}\left(\cos \left(\theta_{\mathrm{r}}\right) \leq h_{\phi_{\mathrm{r}}}\right)=1-F_{\theta_{\mathrm{r}}}\left(\cos ^{-1}\left(h_{\phi_{\mathrm{r}}}\right)\right) .
$$

Subsequently, the PDF of $h_{\phi_{\mathrm{r}}}, f_{h_{\phi_{\mathrm{r}}}}($.$) , is:$

$$
f_{h_{\phi_{\mathrm{r}}}}\left(h_{\phi_{\mathrm{r}}}\right)=\frac{C_{2}}{\sqrt{1-h_{\phi_{\mathrm{r}}}^{2}}} f_{\theta_{\mathrm{r}}}\left(\cos ^{-1}\left(h_{\phi_{\mathrm{r}}}\right)\right),
$$

where $C_{2}$ is a normalization coefficient. Afterwards, the PDF of $h$ can be obtained as:

$$
f_{h}(h)=\int f_{h \mid h_{\phi_{\mathrm{r}}}}\left(h \mid h_{\phi_{\mathrm{r}}}\right) f_{h_{\phi_{\mathrm{r}}}}\left(h_{\phi_{\mathrm{r}}}\right) d h_{\phi_{\mathrm{r}}},
$$

where

$$
\begin{aligned}
& f_{h \mid h_{\phi_{\mathrm{r}}}}\left(h \mid h_{\phi_{\mathrm{r}}}\right)=\frac{1}{h_{\phi_{\mathrm{r}}}} f_{h_{0}}\left(\frac{h}{h_{\phi_{\mathrm{r}}}}\right)
\end{aligned}
$$

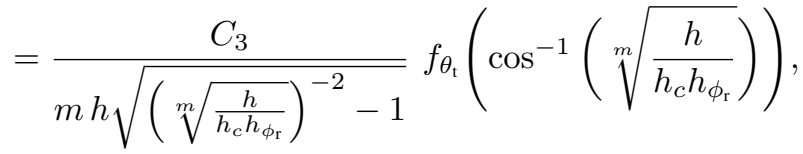

and $C_{3}$ is a normalization coefficient.

\section{Tx-Rx Angular Misalignments and Rx Displacements}

In this case, $\phi_{\mathrm{t}} \neq \theta_{\mathrm{t}}$ and $\phi_{\mathrm{r}} \neq \theta_{\mathrm{r}}$. We assume that the $\mathrm{Rx}$ displacements are negligible, compared to the link range, which is reasonable in practice. Consequently, we assume $D \approx L$, and hence, consider $h_{c}$ as almost constant. Given that we have six RVs that are involved in $h$ (i.e., $x_{\mathrm{r}}, y_{\mathrm{r}}, \varphi_{\mathrm{t}}$, $\varphi_{\mathrm{r}}, \theta_{\mathrm{t}}$, and $\left.\theta_{\mathrm{r}}\right)$, the derivation of the general form of $f_{h}(h)$ is quite complex. Therefore, for simplicity, and similar to the approach considered in [51], we derive the PDF of $h$ by fixing the $\operatorname{Rx}$ position $\left(x_{\mathrm{r}}\right.$ and $\left.y_{\mathrm{r}}\right)$ and the azimuthal angles ${ }^{5} \varphi_{\mathrm{t}}$ and $\varphi_{\mathrm{r}}$. According to (27), we have $\cos \left(\phi_{\mathrm{t}}\right)=a_{\mathrm{t}} \sin \left(\theta_{\mathrm{t}}\right)+b \cos \left(\theta_{\mathrm{t}}\right)$, which can be written as [51]:

$$
\cos \left(\phi_{\mathrm{t}}\right)=\operatorname{sgn}\left(a_{\mathrm{t}}\right) \sqrt{a_{\mathrm{t}}^{2}+b^{2}} \sin \left(\theta_{\mathrm{t}}+\tan ^{-1}\left(b / a_{\mathrm{t}}\right)\right) .
$$

\footnotetext{
${ }^{5}$ As suggested in [53], this would correspond to the case where the Rx only tilts to front and back while the Rx normal $\hat{\boldsymbol{n}}_{r}$ faces the Tx.
}

We consider in the following the case of $a_{\mathrm{t}}<0$, i.e., $\operatorname{sgn}\left(a_{\mathrm{t}}\right)=$ -1 , which allows a simpler derivation ${ }^{6}$ of $f_{h}(h)$. Recall our notation of $h=h_{\mathrm{c}} h_{\phi_{\mathrm{t}}} h_{\phi_{\mathrm{r}}}=h_{0} h_{\phi_{\mathrm{r}}}$. Thus,

$$
f_{h \mid h_{\phi_{\mathrm{r}}}}\left(h \mid h_{\phi_{\mathrm{r}}}\right)=\frac{1}{h_{\phi_{\mathrm{r}}}} f_{h_{0}}\left(\frac{h}{h_{\phi_{\mathrm{r}}}}\right) .
$$

The CDF of $h_{0}$ can be written as:

$$
\begin{aligned}
& F_{h_{0}}\left(h_{0}\right)=\operatorname{Pr}\left(h_{c} \cos ^{m}\left(\phi_{\mathrm{t}}\right) \leq h_{0}\right) \\
& =\operatorname{Pr}\left(\theta_{\mathrm{t}}>-\sin ^{-1}\left(\frac{\sqrt[m]{\frac{h_{0}}{h_{c}}}}{\sqrt{a_{\mathrm{t}}^{2}+b^{2}}}\right)-\tan ^{-1}\left(\frac{b}{a_{\mathrm{t}}}\right)\right) \\
& =1-F_{\theta_{\mathrm{t}}}\left(-\sin ^{-1}\left(\frac{\sqrt[m]{\frac{h_{0}}{h_{c}}}}{\sqrt{a_{\mathrm{t}}^{2}+b^{2}}}\right)-\tan ^{-1}\left(\frac{b}{a_{\mathrm{t}}}\right)\right) .
\end{aligned}
$$

Consequently,

$$
\begin{aligned}
f_{h_{0}}\left(h_{0}\right) & =\frac{-C_{4} \sqrt[m]{\frac{h_{0}}{h_{c}}}}{m h_{0} \sqrt{a_{\mathrm{t}}^{2}+b^{2}-\left(\sqrt[m]{\frac{h_{0}}{h_{c}}}\right)^{2}}} \\
& \times f_{\theta_{\mathrm{t}}}\left(-\sin ^{-1}\left(\frac{\sqrt[m]{\frac{h_{0}}{h_{c}}}}{\sqrt{a_{\mathrm{t}}^{2}+b^{2}}}\right)-\tan ^{-1}\left(\frac{b}{a_{\mathrm{t}}}\right)\right),
\end{aligned}
$$

where $C_{4}$ is a normalization coefficient. Similarly, using (28), we can write $\cos \left(\phi_{\mathrm{r}}\right)=a_{\mathrm{r}} \sin \left(\theta_{\mathrm{r}}\right)+b \cos \left(\theta_{\mathrm{r}}\right)$. Then, the PDF of $h_{\phi_{\mathrm{r}}}$ can be given as:

$$
\begin{aligned}
f_{h_{\phi_{\mathrm{r}}}}\left(h_{\phi_{\mathrm{r}}}\right) & =\frac{-C_{5}}{\sqrt{a_{\mathrm{r}}^{2}+b^{2}-h_{\phi_{\mathrm{r}}}^{2}}} \\
& \times f_{\theta_{\mathrm{r}}}\left(-\sin ^{-1}\left(\frac{h_{\phi_{\mathrm{r}}}}{\sqrt{a_{\mathrm{r}}^{2}+b^{2}}}\right)-\tan ^{-1}\left(\frac{b}{a_{\mathrm{r}}}\right)\right),
\end{aligned}
$$

where $C_{5}$ is a normalization coefficient. Therefore for this case,

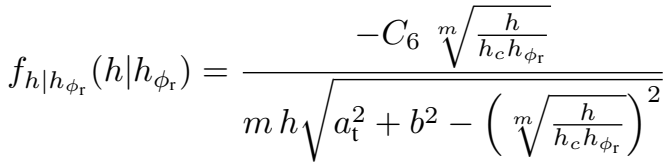

$$
\begin{aligned}
& \times f_{\theta_{\mathrm{t}}}\left(-\sin ^{-1}\left(\frac{\sqrt[m]{\frac{h}{h_{c} h_{\phi_{\mathrm{r}}}}}}{\sqrt{a_{\mathrm{t}}^{2}+b^{2}}}\right)-\tan ^{-1}\left(\frac{b}{a_{\mathrm{t}}}\right)\right),
\end{aligned}
$$

and $C_{6}$ is a normalization coefficient. Lastly, $f_{h}(h)$ is obtained from (38) by marginalization.

\footnotetext{
${ }^{6}$ The PDF of $h$ for the case of $a_{\mathrm{t}}>0$ is much more complex but can be obtained using the proposed approach in [51].
} 
D. Limited Rx FoV; Tx-Rx Angular Misalignments and $R x$ Displacements

Now we apply to the last stage the FoV limitation. Inspired by the approach in [54], the CDF of $h_{\phi_{\mathrm{r}}}$ needs to be modified as follows:

$$
\begin{aligned}
F_{h_{\phi_{\mathrm{r}}}}\left(h_{\phi_{\mathrm{r}}}\right) & =\operatorname{Pr}\left(\cos \left(\phi_{\mathrm{r}}\right) \Pi\left(\frac{\phi_{\mathrm{r}}}{\phi_{\mathrm{FoVr}}}\right) \leq h_{\phi_{\mathrm{r}}}\right) \\
& =\underbrace{\operatorname{Pr}\left(\cos ^{-1}\left(h_{\phi_{\mathrm{r}}}\right)<\phi_{\mathrm{r}} \leq \phi_{\mathrm{FoVr}}\right)}_{1-F_{\phi_{\mathrm{r}}}\left(\cos ^{-1}\left(h_{\phi_{\mathrm{r}}}\right)\right)}+\underbrace{\operatorname{Pr}\left(\phi_{\mathrm{r}}>\phi_{\mathrm{FoVr}}\right)}_{1-F_{\phi_{\mathrm{r}}}\left(\phi_{\mathrm{FoV} \mathrm{r}}\right)} \\
& =1-F_{\theta_{\mathrm{r}}}\left(-\sin ^{-1}\left(\frac{h_{\phi_{\mathrm{r}}}}{\sqrt{a_{\mathrm{r}}^{2}+b^{2}}}\right)-\tan ^{-1}\left(\frac{b}{a_{\mathrm{r}}}\right)\right) \\
& +\left(1-F_{\phi_{\mathrm{r}}}\left(\phi_{\mathrm{FoVr}}\right)\right) .
\end{aligned}
$$

In the right side of the first line of (46), the first term accounts for the probability that an incident ray falls within the Rx FoV, while the second terms corresponds to a link interruption. The resulting PDF is then:

$$
\begin{aligned}
f_{h_{\phi_{\mathrm{r}}}}\left(h_{\phi_{\mathrm{r}}}\right) & =\frac{-C_{7}}{\sqrt{a_{\mathrm{r}}^{2}+b^{2}-h_{\phi_{\mathrm{r}}}^{2}}} \\
& \times f_{\theta_{\mathrm{r}}}\left(-\sin ^{-1}\left(\frac{h_{\phi_{\mathrm{r}}}}{\sqrt{a_{\mathrm{r}}^{2}+b^{2}}}\right)-\tan ^{-1}\left(\frac{b}{a_{\mathrm{r}}}\right)\right) \\
& +\left(1-F_{\phi_{\mathrm{r}}}\left(\phi_{\mathrm{FoV}}\right)\right) \delta\left(h_{\phi_{\mathrm{r}}}\right)
\end{aligned}
$$

where the Dirac delta function accounts for the discontinuity of the CDF at $h_{\phi_{\mathrm{r}}}=0$ [54], and $C_{7}$ is a normalization coefficient. The PDF of $h$ can be obtained by marginalization from (38) using (45) and (47).

\section{Numerical RESUlts}

This section presents numerical results to study the link performance and the impact of link parameter optimization.

\section{A. Parameter Specification}

We consider a vertical link operating at depth $Z$ in clear ocean waters having a chlorophyll concentration of $C_{\mathrm{cl}}=$ $0.5 \mathrm{mg} / \mathrm{m}^{3}$. At the Tx, we use a blue LED of wavelength $470 \mathrm{~nm}$ with a spectral half-width of $20 \mathrm{~nm}$ [55]. For this wavelength and the considered $C_{\mathrm{cl}}, K_{\mathrm{d}}$ is approximately $0.08 \mathrm{~m}^{-1}$ [56], [57]. At the Rx, we use an optical filter of BW $\Delta \lambda=20 \mathrm{~nm}$, where for the sake of simplicity, we assume that its signal transmission $T_{\mathrm{s}}$ is nearly constant and equal to 1 [58]. Also, a blue-sensitive SiPM with reference SensL B-series MicroSB 30020 [59] is considered. Note that the deadtime of the SiPM is $\tau_{d}=90 \mathrm{~ns}$, during which the detector is unable to detect a new incoming photon [20], [60]. The extinction ratio for OOK Off/On symbols is set to $\xi=0.4$ and the bit rate to $R_{b}=1 \mathrm{Mbps}$. It is worth mentioning that $\tau_{d}$ is much smaller than the considered symbol duration, i.e., $1 \mu \mathrm{s}$, and furthermore, the typical modulation BWs of the LED and the SiPM are assumed to be much higher than the signal BW [37]. Hence, no inter-symbol interference affects the transmitted signal. ${ }^{7}$ Unless otherwise specified, the link range (i.e., the AUV operational depth in our case study) is set to $100 \mathrm{~m}$, and the average Tx power is set to $P_{\mathrm{Tx}}=20 \mathrm{~W}$ that can be obtained by using multiple LEDs at the Tx [36], [37]. Table I summarizes the considered parameters used for the link performance evaluation.

We do not consider any forward error correction (FEC) and set the target BER to the FEC threshold $\mathrm{BER}_{\mathrm{th}}=2 \times 10^{-3}$ [63]. To calculate $P_{\text {out }}$, we use statistical and analytical approaches. By the statistical approach, in the general case of misaligned link illustrated in Fig. 4, for instance, we generate independent RVs $\theta_{\mathrm{t}}, \phi_{\mathrm{t}}, \varphi_{\mathrm{t}}, \theta_{\mathrm{r}}, \phi_{\mathrm{r}}, \varphi_{\mathrm{r}}, x_{\mathrm{r}}$, and $y_{\mathrm{r}}$ according to the considered distributions, and use them to calculate the corresponding channel attenuation $h$. Then, for each channel realization $h$, the BER is calculated from (11). The outage probability $P_{\text {out }}$ is calculated based on more than $10^{7}$ channel realizations and for a given $\mathrm{BER}_{\mathrm{th}}$. On the other hand, by the analytical method, we calculate the approximate $P_{\text {out }}$ from (14) using the derived analytical PDFs $f_{h}(h)$ in Section IV, through numerical integration [64].

Concerning the parameters related to angular misalignment and displacement of the AUV, we consider a range of a few degrees for $\left(\sigma_{\theta \mathrm{t}}, \sigma_{\theta \mathrm{r}}\right)$ and a range of a few meters for $\sigma_{\mathrm{pr}}$, which are close to practical uplink and downlink scenarios. ${ }^{8}$

In what follows, we first illustrate the effect of different factors that result in pointing errors in the considered case studies. Then, we focus on $\mathrm{Tx} / \mathrm{Rx}$ parameter optimization, i.e., $m$ and $\theta_{\mathrm{FoV}}$, and investigate its effect on the overall link performance.

\section{B. Effect of Misalignment Parameters on Outage Probability Buoy-to-AUV Downlink}

First, to get an idea of the effect of wind speed and AUV angular misalignments and displacements, consider the downlink configuration, i.e., from the buoy to the AUV, and see the impact of different parameters. Consider a (fixed) LED Lambertian order of $m=20$, corresponding to a beam divergence of about $15^{\circ}$, a (fixed) large $\operatorname{Rx} \mathrm{FoV} \phi_{\mathrm{FoVr}}=\pi / 2$, and a link range of $65 \mathrm{~m}$. For the three cases specified in Subsections IV-A, IV-B, and IV-C, are presented in Fig. 5 the outage probability versus the corresponding misalignment parameter, i.e., $U, \sigma_{\theta \mathrm{r}}$, and $\sigma_{\mathrm{pr}}$, respectively.

Notice the good match between the simulation-based (statistical MC) and analytical results, which shows the accuracy of the approximate analytical formulas. Indeed, they provide a tight lower bound on $P_{\text {out }}$ for the considered operating conditions. From Fig. 5(a), for fixed (and exact) AUV position

\footnotetext{
${ }^{7}$ For very high data rates where the deadtime limitation induces intersymbol interference, [61] proposed an optimal signal detection approach, based on the number of counts and arrival times of the photons. Also, appropriate signaling schemes were proposed in [37], [62] for the case of limited $\mathrm{Tx} / \mathrm{Rx}$ modulation $\mathrm{BW}$ with respect to the data rate.

${ }^{8}$ For instance, some ultra-short baseline (USBL) underwater acoustic positioning systems used for AUVs, such as the Subsonus USBL and its corresponding transponder, Subsonus tag, have their respective pitch and roll accuracies as $\sim 0.1^{\circ}$ and $\sim 1^{\circ}$ [65], [66]. Also, the position accuracies of Subsonus USBL and Subsonus tag are around 0.5 and $1.5 \mathrm{~m}$, respectively, at a typical range of $100 \mathrm{~m}[65],[66]$.
} 
TABLE I

Simulation Parameters

\begin{tabular}{|l|l|}
\hline Parameter & Value \\
\hline \hline Tx wavelength $\lambda$ & $470 \mathrm{~nm}$ \\
\hline Tx average transmit power $P_{\mathrm{Tx}}$ & $20 \mathrm{~W}$ \\
\hline OOK extinction ratio $\xi$ & 0.4 \\
\hline Bit rate $R_{\mathrm{b}}$ & $1 \mathrm{Mbps}$ \\
\hline Optical filter BW & $20 \mathrm{~nm}$ \\
\hline SiPM active area $A_{\mathrm{PD}}$ & $9 \mathrm{~mm}^{2}$ \\
\hline SiPM, number of SPADs & 10998 \\
\hline SiPM, fill factor & $48 \%$ \\
\hline SiPM gain $G$ & $10^{6}$ \\
\hline SiPM photon detection efficiency $\Upsilon_{\mathrm{PDE}}$ & $24 \%$ \\
\hline SiPM dark count rate $f_{\mathrm{DCR}}$ & $6.6 \mathrm{MHz}$ \\
\hline SiPM dark current $I_{\mathrm{d}}$ & $1.10 \mu \mathrm{A}$ \\
\hline SiPM prob. of cross-talk $P_{\mathrm{CT}}$ & $0.03 \%$ \\
\hline SiPM prob. of after-pulsing $P_{\mathrm{AP}}$ & $0.2 \%$ \\
\hline SiPM deadtime $\tau_{d}$ & $90 \mathrm{~ns}$ \\
\hline SiPM excess noise factor $F$ & 1.1 \\
\hline TIA load resistance $R_{\mathrm{L}}$ & $1 \mathrm{k} \Omega$ \\
\hline Target BER, BER & $2 \times 10^{-3}$ \\
\hline
\end{tabular}

and orientation, notice a significant impact of the wind speed $U$ on the link performance. Comparing this with Fig. 5(b), notice that AUV angular misalignments have a less significant impact on $P_{\text {out }}$; it is almost unaffected for $\sigma_{\theta \mathrm{r}}$ up to $10^{\circ}$ corresponding to little angular deviations of the $\mathrm{Rx}$ from the beam LoS. For angular deviations larger than $\sim 15^{\circ}$ (which is close to the considered Tx beam divergence), a significant $P_{\text {out }}$ degradation is noticed as the Rx largely turns away from the beam spot.

For Fig. 5(c), following our approach in Subsection IV-C for the derivation of the analytical PDF of $h$, we have set $\varphi_{\mathrm{t}}=\varphi_{\mathrm{r}}=30^{\circ}$, and calculated $P_{\text {out }}$ for a fixed displacement $x_{\mathrm{r}}=y_{\mathrm{r}}$. Notice a significant performance degradation with displacement error. For $x_{\mathrm{r}}=y_{\mathrm{r}} \gtrsim 5 \mathrm{~m}, P_{\text {out }}$ is almost equal to one, meaning that the $\mathrm{Rx}$ is completely away from the transmitted beam LoS.

Similar to Fig. 5(a), we have further presented in Fig.6 $P_{\text {out }}$ as a function of the average transmit power $P_{\mathrm{Tx}}$ and the sea surface wind speed $U$, assuming exactly positioned $\mathrm{Rx}$. We notice that with increase in $U$, an increase in $P_{\mathrm{Tx}}$ can only marginally compensate for this $P_{\text {out }}$ degradation. However, it will be shown in the following subsection that the optimization of the $\mathrm{Tx} / \mathrm{Rx}$ parameters can improve the link performance considerably.

\section{Effect of Tx/Rx Parameter Optimization, Buoy-to-AUV Downlink}

For different link conditions, we investigate the effect of optimizing Tx and Rx parameters, i.e., $m$ and $\phi_{\mathrm{FoVr}}$ for limited Rx FoV. Two scenarios are considered: without and with $\mathrm{Rx}$ position displacement. The presented results are obtained using $\mathrm{MC}$ simulations.

1) Scenario 1: Negligible Rx position displacement: Consider first the case where there is no position displacement for the Rx, see Fig. 3. Figure 7 shows the 3D plot of $P_{\text {out }}$ versus $m$ and $\phi_{\mathrm{FoVr}}$ for $U=3 \mathrm{~m} / \mathrm{s}$ and $\sigma_{\theta \mathrm{r}}=5^{\circ}$. We notice that significant performance improvement can be obtained by

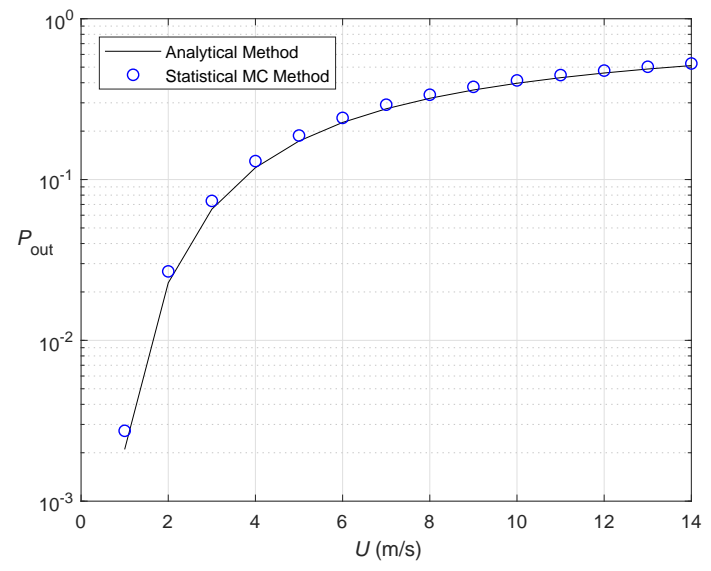

(a) Tx angular misalignments and fixed $\mathrm{Rx}$

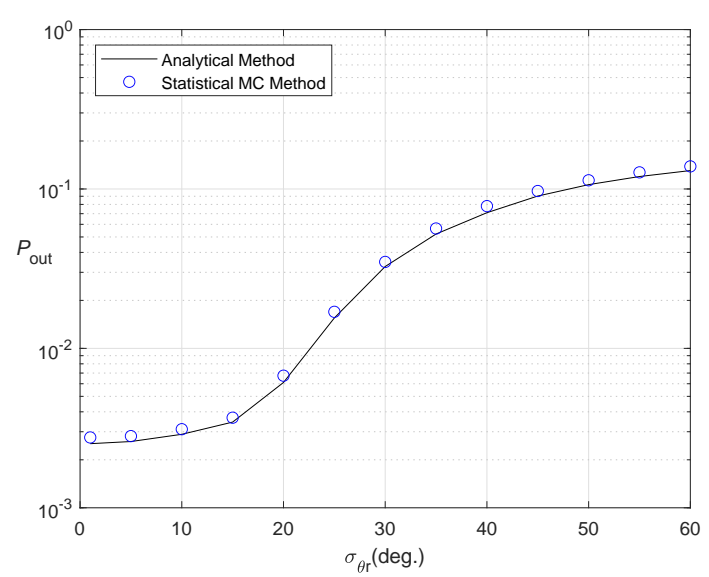

(b) Tx-Rx angular misalignments and fixed $\mathrm{Rx}$

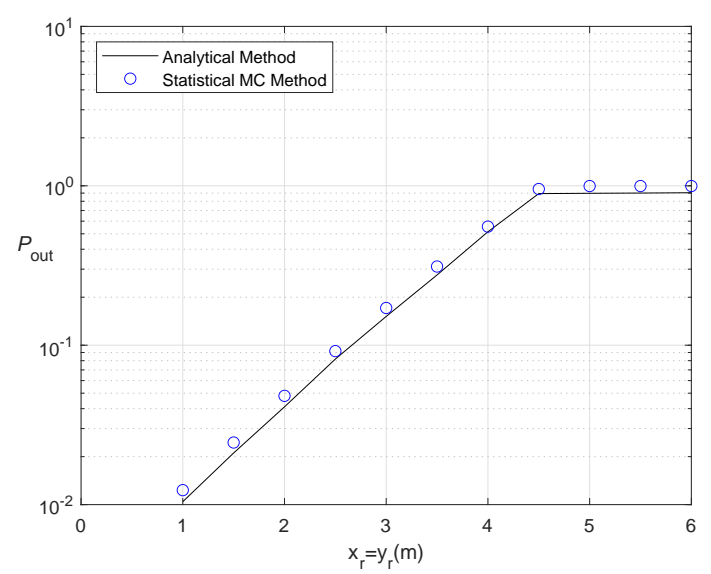

(c) Tx-Rx angular misalignments and Rx displacements

Fig. 5. Effect of misalignment parameters on $P_{\text {out }}$ performance using the analytical and statistical MC approaches; Downlink transmission with $m=20, P_{\mathrm{Tx}}=20 \mathrm{~W}, \xi=0.4, \phi_{\mathrm{FoVr}}=\pi / 2$, and $Z=65 \mathrm{~m}$; (a) $\sigma_{\theta \mathrm{r}}=0, \sigma_{\mathrm{pr}}=0$; (b) $U=1 \mathrm{~m} / \mathrm{s}, \sigma_{\mathrm{pr}}=0$; (c) $U=1 \mathrm{~m} / \mathrm{s}, \sigma_{\theta \mathrm{r}}=5^{\circ}$. 


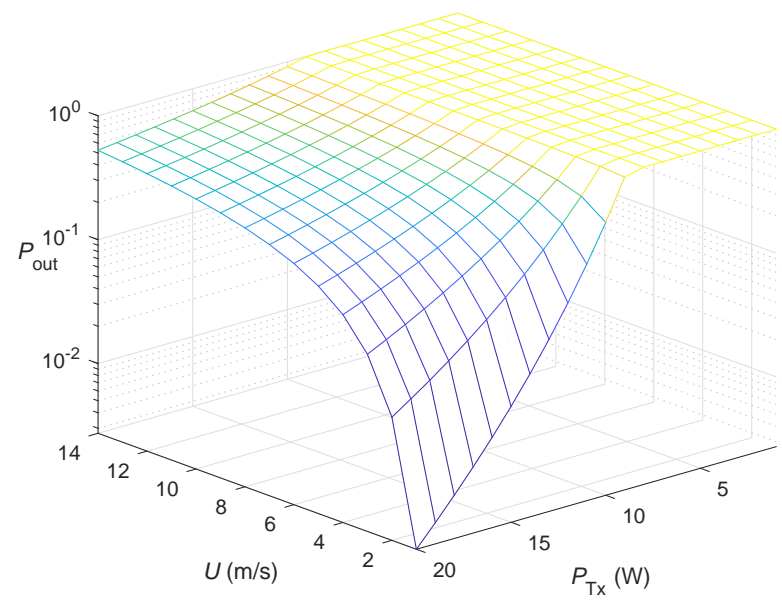

Fig. 6. Effect of wind speed $U$ and the average transmit power $P_{\text {Tx }}$ on the $P_{\text {out }} ; \sigma_{\theta \mathrm{r}}=0, \sigma_{\mathrm{pr}}=0, m=20, \xi=0.4, \phi_{\mathrm{FoVr}}=\pi / 2$, and $Z=65 \mathrm{~m}$.

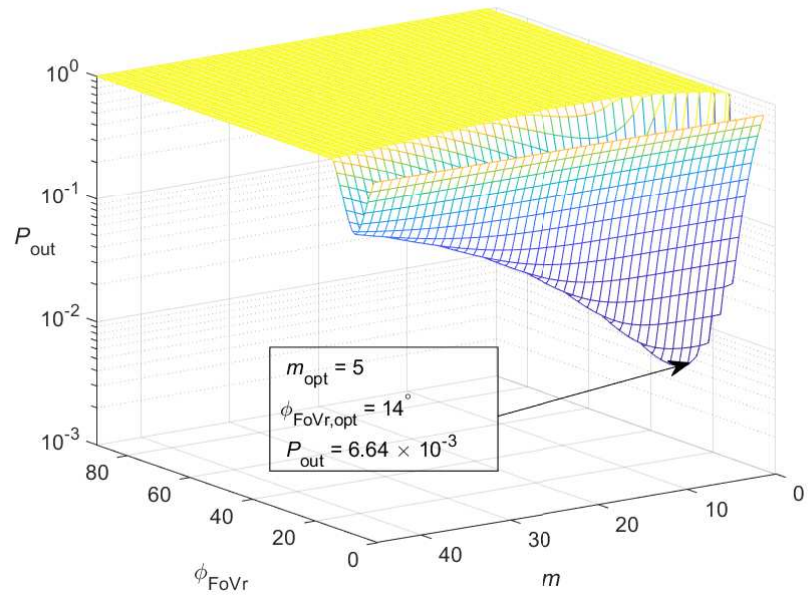

Fig. 7. Scenario 1: Effect of $m$ and $\phi_{\mathrm{FoVr}}$ (in degrees) on $P_{\text {out }}$ for $U=3 \mathrm{~m} / \mathrm{s}$ and $\sigma_{\theta \mathrm{r}}=5^{\circ} . Z=100 \mathrm{~m}, P_{\mathrm{Tx}}=20 \mathrm{~W}$.

using the optimal parameters in terms of reducing the outage probability, here, $m_{\mathrm{opt}} \approx 5$ and $\phi_{\mathrm{FoVr}, \mathrm{opt}} \approx 14^{\circ}$ resulting in the minimum $P_{\text {out }}=6.64 \times 10^{-3}$.

Subsequently, for increased wind speeds of $U=6$ and $9 \mathrm{~m} / \mathrm{s}$ and $\sigma_{\theta \mathrm{r}}=5^{\circ}$, we have shown in Fig. 8 plots of $P_{\text {out }}$ versus $m$. To simplify the presentation in a $2 \mathrm{D}$ plot, we have considered for each $m$ the corresponding $\phi_{\text {FoVr,opt }}$ resulting in the minimum $P_{\text {out }}$. As reference to the results in Fig.7, we have also shown the $P_{\text {out }}$ plot for $U=3 \mathrm{~m} / \mathrm{s}$. These results clearly show the destructive impact of $U$ on the link performance. Also, optimized Tx-Rx parameters allow a significant improvement in the link performance; the optimum parameters and the corresponding $P_{\text {out }}$ values are indicated in the figure. We notice that for increased $U$, a smaller $m$ should be used. This is quite rational, since by increased Tx angular misalignments, we should use a wider Tx beam to minimize the probability of receiving a too weak signal at the Rx. In other words, surface waves are more likely to direct the signal

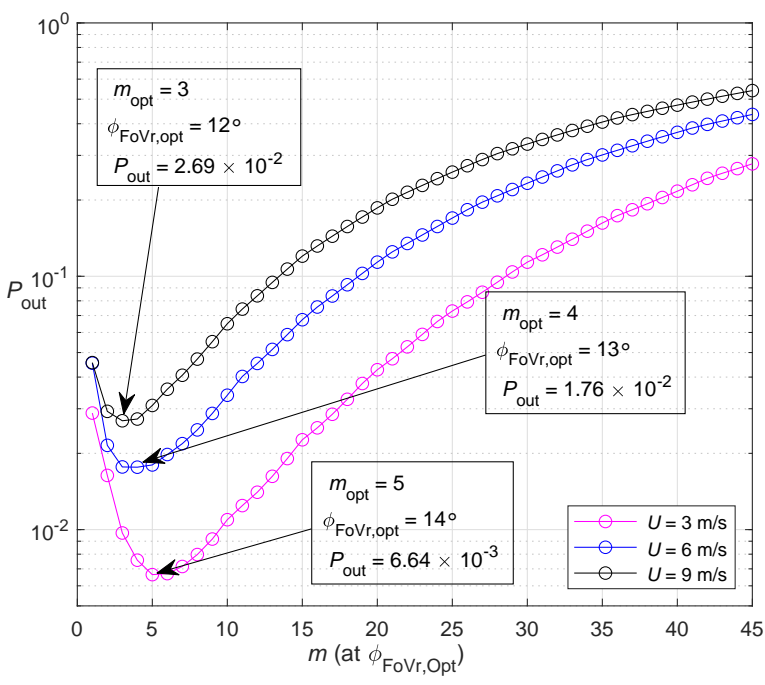

Fig. 8. Scenario 1: Effect of $m$ on $P_{\text {out }}$ using optimum $\phi_{\mathrm{FoVr}}$ for $U=3,6$, and $9 \mathrm{~m} / \mathrm{s}$ and $\sigma_{\theta \mathrm{r}}=5^{\circ} . Z=100 \mathrm{~m}, P_{\text {Tx }}=20 \mathrm{~W}$.

TABLE II

SCENARIO 1 (DOWNLINK): OPTIMUM PARAMETERS FOR DIFFERENT $\sigma_{\theta \mathrm{r}}$ OF AUV RX DISORIENTATION; $U=3 \mathrm{~m} / \mathrm{s}$, $Z=100 \mathrm{~m}, P_{\mathrm{Tx}}=20 \mathrm{~W}$.

\begin{tabular}{|c||c||c||c||c|}
\hline$\sigma_{\theta \mathrm{r}}($ deg. $)$ & $m_{\text {opt }}$ & $\theta_{\text {FoVr,opt }}($ deg. $)$ & $P_{\text {out }}$ & $P_{\text {int }}$ \\
\hline 5 & 5 & 14 & $6.64 \times 10^{-3}$ & $5.1 \times 10^{-3}$ \\
\hline 7 & 6 & 15 & $3.84 \times 10^{-2}$ & $3 \times 10^{-2}$ \\
\hline 10 & 8 & 16 & $1.17 \times 10^{-1}$ & $8.9 \times 10^{-2}$ \\
\hline
\end{tabular}

away from the Rx FoV, thus the beam should be widened to insure the signal reaches the Rx. The optimum Rx FoV values are rather close; yet, $\phi_{\mathrm{FoVr}, \mathrm{opt}}$ decreases by increasing $U$ : it equals $14^{\circ}, 13^{\circ}$, and $12^{\circ}$, for $U=3,6$, and $9 \mathrm{~m} / \mathrm{s}$, respectively. To explain this, we note from (1) and (2) that the effective $\mathrm{Rx}$ area $A_{\mathrm{eff}}$ is inversely related to $\phi_{\mathrm{FoVr}}$ due to the use of a non-imaging concentrator. Hence, a decrease in $\phi_{\mathrm{FoVr}, \mathrm{opt}}$ arising from an increased $U$ is justified by achieving a larger effective light collection area, compensating for more pointing errors.

To see the effect of the Rx's $\sigma_{\theta \mathrm{r}}$, we have fixed $U$ at $3 \mathrm{~m} / \mathrm{s}$ and increased $\sigma_{\theta \mathrm{r}}$ to $7^{\circ}$ and $10^{\circ}$. Results are summarized in Table II, where we have also provided the corresponding values of $P_{\text {int }}$. From these results, we notice a rather low sensitivity of $m_{\mathrm{opt}}$ and $\theta_{\mathrm{FoVr}, \text { opt }}$ to $\sigma_{\theta \mathrm{r}}$. Interestingly, as $\sigma_{\theta \mathrm{r}}$ increases, the beam needs to be more directive ( $m$ should be increased) and the Rx FoV should increase. Indeed, increasing $m$ increases the directivity of the transmit beam towards the normal of the Rx, while the increase in the $\mathrm{Rx}$ FoV aims to improve signal reception for larger $\mathrm{Rx}$ deviations.

Let us now investigate the effect of the average transmit power on the parameter optimization. We have summarized the results in Table III, where we notice that by decrease in $P_{\mathrm{Tx}}, m_{\mathrm{opt}}$ is increased, i.e., the beam is made more directive to reduce the effect of geometric loss, while $\phi_{\mathrm{FoVr}, \mathrm{opt}}$ is decreased to increase the Rx effective area of light collection. Overall, these two factors compensate for the reduced signal-to-noise 
TABLE III

SCENARIO 1 (DOWNLINK): OPTIMUM PARAMETERS FOR DIFFERENT $P_{\mathrm{Tx}} ; U=3 \mathrm{~m} / \mathrm{s}, \sigma_{\theta \mathrm{r}}=5^{\circ}, Z=100 \mathrm{~m}$.

\begin{tabular}{|c||c||c||c||c|}
\hline$P_{\text {Tx }}(\mathrm{W})$ & $m_{\text {opt }}$ & $\phi_{\text {FoVr,opt }}($ deg. $)$ & $P_{\text {out }}$ & $P_{\text {int }}$ \\
\hline 30 & 4 & 16 & $1.5 \times 10^{-3}$ & $1.4 \times 10^{-3}$ \\
\hline 20 & 5 & 14 & $6.64 \times 10^{-3}$ & $5.1 \times 10^{-3}$ \\
\hline 10 & 7 & 11 & $4.67 \times 10^{-2}$ & $2.78 \times 10^{-2}$ \\
\hline
\end{tabular}

TABLE IV

SCENARIO 1 (DOWNLINK): OPTIMUM PARAMETERS FOR DIFFERENT LINK SPANS $Z ; U=3 \mathrm{~m} / \mathrm{s}, \sigma_{\theta \mathrm{r}}=5^{\circ}, P_{\mathrm{Tx}}=20 \mathrm{~W}$.

\begin{tabular}{|c||c||c||c||c|}
\hline$Z(\mathrm{~m})$ & $m_{\text {opt }}$ & $\theta_{\text {FoV }, \text { opt }}($ deg. $)$ & $P_{\text {out }}$ & $P_{\text {int }}$ \\
\hline 90 & 3 & 20 & $3.39 \times 10^{-5}$ & $3.1 \times 10^{-5}$ \\
\hline 100 & 5 & 14 & $6.64 \times 10^{-3}$ & $5.1 \times 10^{-3}$ \\
\hline 110 & 6 & 9 & $7.81 \times 10^{-2}$ & $7.17 \times 10^{-2}$ \\
\hline
\end{tabular}

ratio (SNR) with decrease in $P_{\mathrm{Tx}}$. We have further presented in Table IV the results for different link ranges. Logically, the trend in $m_{\mathrm{opt}}$ and $\phi_{\mathrm{FoVr}, \mathrm{opt}}$ with increase in $Z$ is the same as with decrease in $P_{\mathrm{Tx}}$. We notice that, as the Tx-Rx distance increases, $m_{\mathrm{opt}}$ is increased while $\phi_{\mathrm{FoV}, \mathrm{opt}}$ is decreased.

2) Scenario 2: Considering Rx position displacement: Let us now include in the considered link configuration the deviations in the position of the AUV Rx with respect to the buoy Tx. We have presented in Table $\mathrm{V}$ the results for $U=3 \mathrm{~m} / \mathrm{s}$, $\sigma_{\theta \mathrm{r}}=5^{\circ}$, and different values of the Rx displacement standard deviation $\sigma_{\mathrm{pr}}$. The case of $\sigma_{\mathrm{pr}}=0$ corresponds to the results previously presented in Fig. 7. Reasonably, with an increase in $\sigma_{\mathrm{pr}}$, the corresponding $P_{\text {out }}$ and $P_{\text {int }}$ increase. For more important displacements, i.e., higher $\sigma_{\mathrm{pr}}$, we need to widen the Tx beam (by decreasing $m$ ) and increase the effective Rx area (by decreasing $\phi_{\mathrm{FoVr}}$ ). Meanwhile, notice the small change in the optimum parameters $m_{\mathrm{opt}}$ and $\phi_{\mathrm{FoV} \text { ropt }}$ with increase in $\sigma_{\mathrm{pr}}$. This can be explained by the fact that, given the Tx beam divergence and the link range, the Rx displacements are not significant compared to the beam spot radius. This latter is considered as the radius where we get the half intensity with respect to the beam center, which is around $30 \mathrm{~m}$ for $m=5$, for instance.

\section{Effect of Tx/Rx Parameter Optimization, AUV-to-Buoy Up- link}

For the case of uplink transmission, i.e., from the AUV to the buoy, the angular misalignments are modeled by $\sigma_{\theta \mathrm{t}}$ (for

TABLE V

SCENARIO 2 (DOWNLINK): OPTIMUM PARAMETERS FOR DIFFERENT $\sigma_{\mathrm{pr}}$ OF AUV RX DISPLACEMENTS; $U=3 \mathrm{~m} / \mathrm{s}, \sigma_{\theta \mathrm{r}}=5^{\circ}$ $Z=100 \mathrm{~m}, P_{\mathrm{Tx}}=20 \mathrm{~W}$.

\begin{tabular}{|c||c||c||c||c|}
\hline$\sigma_{\mathrm{pr}}(\mathrm{m})$ & $m_{\text {opt }}$ & $\theta_{\mathrm{FoVr}, \text { opt }}($ deg. $)$ & $P_{\text {out }}$ & $P_{\text {int }}$ \\
\hline 0 & 5 & 14 & $6.64 \times 10^{-3}$ & $5.1 \times 10^{-3}$ \\
\hline 2 & 5 & 14 & $1.02 \times 10^{-2}$ & $6.5 \times 10^{-3}$ \\
\hline 6 & 4 & 13 & $4.66 \times 10^{-2}$ & $4.03 \times 10^{-2}$ \\
\hline 12 & 4 & 13 & $2.8 \times 10^{-1}$ & $2.33 \times 10^{-1}$ \\
\hline
\end{tabular}

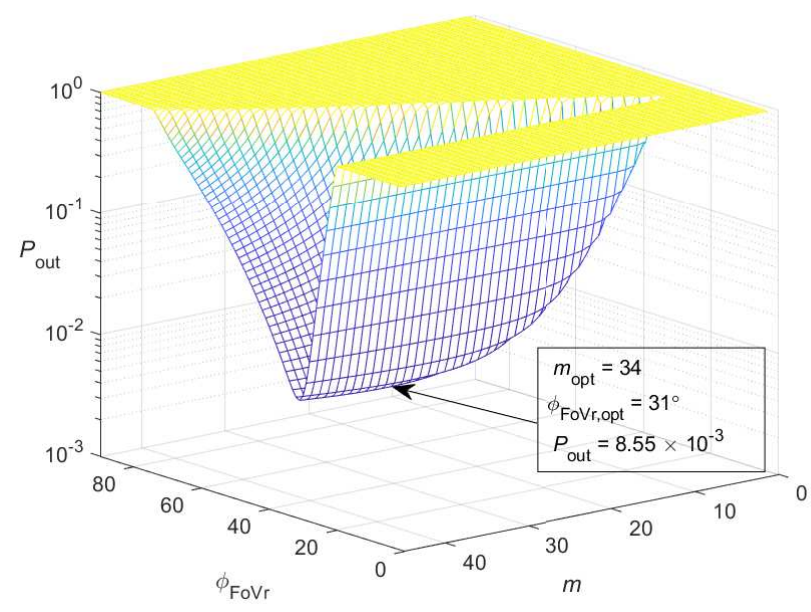

Fig. 9. Scenario 3: Effect of $m$ and $\phi_{\mathrm{FoVr}}$ on $P_{\mathrm{out}}$ for the uplink. AUV's Tx with $\sigma_{\theta \mathrm{t}}=5^{\circ}$ and Buoy's Rx with $U=3 \mathrm{~m} / \mathrm{s} . Z=100 \mathrm{~m}, P_{\mathrm{Tx}}=20 \mathrm{~W}$.

the $\mathrm{Tx}$ ) and $U$ (for the $\mathrm{Rx}$ ), respectively.

1) Scenario 3: Negligible Tx position displacement: Figure 9 shows the 3D plots of $P_{\text {out }}$ versus $m$ and $\phi_{\mathrm{FoVr}}$ for $\sigma_{\theta \mathrm{t}}=5^{\circ}$ at the Tx and $U=3 \mathrm{~m} / \mathrm{s}$ at the Rx side. The optimum parameters $^{9}$ are $m_{\mathrm{opt}}=34$ and $\phi_{\mathrm{FoVr}, \mathrm{opt}}=31^{\circ}$, resulting in the minimum achievable $P_{\text {out }}=8.55 \times 10^{-3}$.

Similar to Subsection V-C, keeping $U$ at $3 \mathrm{~m} / \mathrm{s}$, we have presented in Fig. 10 the 2D plots of $P_{\text {out }}$ versus $m$ for $\sigma_{\theta \mathrm{t}}=5^{\circ}$, $7^{\circ}$, and $10^{\circ}$. For each $m, \phi_{\mathrm{FoVr}}$ is selected so as to result in the minimum $P_{\text {out }}$. Like for the downlink case in the previous subsection, as $\sigma_{\theta \mathrm{t}}$ is increased (from $5^{\circ}$ to $7^{\circ}$ and $10^{\circ}$ ), the beam needs to be widened (i.e., $m_{\mathrm{opt}}$ decreases from 34 to 30 , and 26), and the effective area of light collection should be increased (i.e., $\phi_{\mathrm{FoVr} \text {,opt }}$ decreases from $31^{\circ}$ to $29^{\circ}$, and $27^{\circ}$ ). Once again, the Tx-Rx parameter optimization trades off geometric loss (related to beam divergence) with the Rx concentrator gain (related to the Rx FoV).

To see better the impact of $U$, we set $\sigma_{\theta \mathrm{t}}$ to $5^{\circ}$ and vary $U$ from 3 to 6 and $9 \mathrm{~m} / \mathrm{s}$. The optimum parameters together with the minimum $P_{\text {out }}$ and $P_{\text {int }}$ are given in Table VI. We notice that for increased $U$, the respective $m_{\mathrm{opt}}$ and $\phi_{\mathrm{FoV} \text {,opt }}$ increase as well, which is consistent with the results presented for Scenario 1 in the previous subsection: The increase in the $\mathrm{Rx}$ angular misalignments is compensated by the increase in $m$ (to make the beam more directive, and hence reduce the geometric loss), and an increase in $\phi_{\mathrm{FoVr}}$ (to improve signal reception for larger Rx deviations although this also results in a smaller concentrator gain).

2) Scenario 4: Considering $T x$ position displacement: Including now into the link configuration the position displacements of the AUV Tx, Table VII shows the optimum parameters and the performance metrics for $U=3 \mathrm{~m} / \mathrm{s}$, $\sigma_{\theta \mathrm{t}}=5^{\circ}$, and four values of $\sigma_{\mathrm{pt}}=0,2,6$, and $12 \mathrm{~m}$. Notice the

\footnotetext{
${ }^{9}$ Note that the larger values of optimum parameters, compared to those of the previously discussed downlink, is due to the difference in the Tx distribution, and subsequently the parameters affecting their angular misalignment.
} 


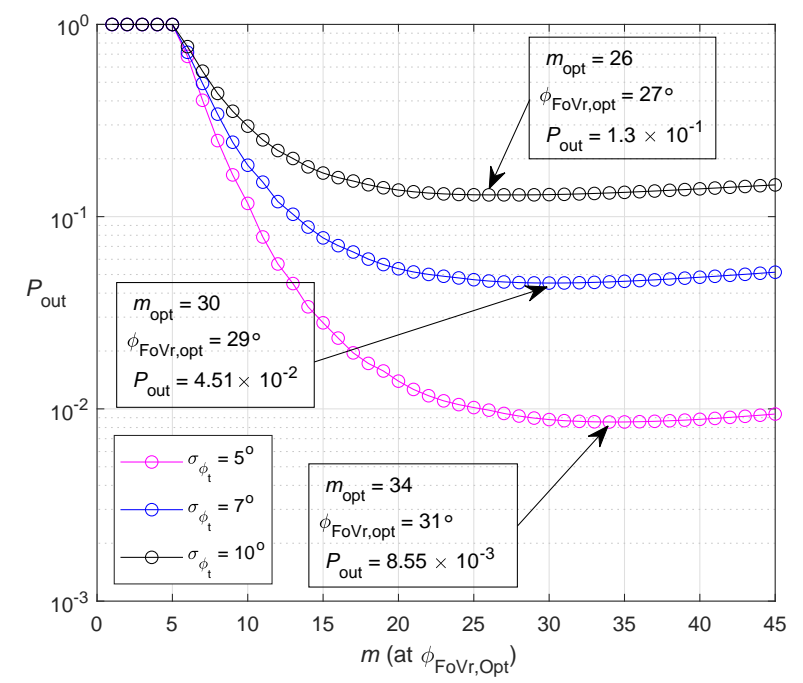

Fig. 10. Scenario 3: Effect of $m$ for an optimum $\phi_{\text {FoVr }}$ on $P_{\text {out }}$ for AUV's Tx $\sigma_{\theta \mathrm{t}}$ of $5^{\circ}, 7^{\circ}$, and $10^{\circ}$ at Buoy's Rx $U=3 \mathrm{~m} / \mathrm{s} . Z=100 \mathrm{~m}, P_{\mathrm{Tx}}=20 \mathrm{~W}$.

TABLE VI

SCENARIO 3 (UPLINK): OPTIMUM PARAMETERS FOR DIFFERENT WIND SPEEDS $U$ AFFECTING THE BUOY RX. NEGLIGIBLE TX (AUV) DISPLACEMENT; $\sigma_{\theta \mathrm{t}}=5^{\circ} . Z=100 \mathrm{~m}, P_{\mathrm{Tx}}=20 \mathrm{~W}$

\begin{tabular}{|c||c||c||c||c|}
\hline$U(\mathrm{~m} / \mathrm{s})$ & $m_{\text {opt }}$ & $\theta_{\mathrm{FoVr}, \text { opt }}($ deg. $)$ & $P_{\text {out }}$ & $P_{\text {int }}$ \\
\hline 3 & 34 & 31 & $8.55 \times 10^{-3}$ & $2 \times 10^{-3}$ \\
\hline 6 & 44 & 35 & $2.05 \times 10^{-2}$ & $5 \times 10^{-3}$ \\
\hline 9 & 45 & 38 & $3.34 \times 10^{-2}$ & $7.7 \times 10^{-3}$ \\
\hline
\end{tabular}

TABLE VII

SCENARIO 4 (UPLINK): OPTIMUM PARAMETERS FOR DIFFERENT AUV TX DISPLACEMENT STANDARD DEVIATIONS $\sigma_{\mathrm{pt}} ; \sigma_{\theta \mathrm{t}}=5^{\circ}$, $U=3 \mathrm{~m} / \mathrm{s} . Z=100 \mathrm{~m}, P_{\mathrm{Tx}}=20 \mathrm{~W}$.

\begin{tabular}{|c||c||c||c||c|}
\hline$\sigma_{\mathrm{pt}}(\mathrm{m})$ & $m_{\text {opt }}$ & $\theta_{\mathrm{FoVr}, \text { opt }}($ deg. $)$ & $P_{\text {out }}$ & $P_{\text {int }}$ \\
\hline 0 & 34 & 31 & $8.55 \times 10^{-3}$ & $2 \times 10^{-3}$ \\
\hline 2 & 37 & 32 & $1.29 \times 10^{-2}$ & $2.2 \times 10^{-3}$ \\
\hline 6 & 40 & 33 & $5.8 \times 10^{-2}$ & $1.28 \times 10^{-2}$ \\
\hline 12 & 43 & 34 & $3.15 \times 10^{-1}$ & $1.19 \times 10^{-1}$ \\
\hline
\end{tabular}

relatively close optimum parameters for the considered cases. This can be justified by the fact that such small displacements impact negligibly the signal reception, given the relatively large size of the beam spot at the Rx side (which is around $17.5 \mathrm{~m}$ for $m=34$, for instance; see also Subsection V-C2). In fact, with increase in $\sigma_{\mathrm{pt}}, m_{\mathrm{opt}}$ and $\phi_{\mathrm{FoVr}, \mathrm{opt}}$ should increase to make the beam more directional on one hand (to decrease the geometric loss), and increase the Rx FoV on the other hand (to improve signal reception). For instance, by increasing $\sigma_{\mathrm{pt}}$ from 0 to $2 \mathrm{~m}, m_{\mathrm{opt}}$ and $\phi_{\mathrm{FoVr}, \text { opt }}$ are increased from 34 to 37 , and from $31^{\circ}$ to $32^{\circ}$, respectively.

\section{CONCLUSIONS}

This paper studies the design of a bi-directional UWOC link between the sea surface and a mobile underwater unit. The presented analysis revealed that a substantial improvement in the link performance can be obtained by optimizing the main link parameters, namely the Tx Lambertian order and the Rx FoV. The corresponding optimal parameters depend on the channel and link deployment parameters including the seasurface wind speed, AUV stability and positioning accuracy, and the received power. Note that the received power depends on the the link range (here, the operation depth of the AUV) and the transmit power, for which the dependence of the optimal parameters was shown.

In practice, these link parameters can be estimated either at the $\mathrm{Tx}$ or at the Rx and be shared with the other side of the link in real time in order to adapt Tx-Rx parameters. This assumes, of course, a bi-directional transmission between the two ends of the link. Note that in the case that one of the terminals is not equipped with an optical transmitter, sending the estimated channel parameters to the other side of the link can be done using an acoustic link given the corresponding required low data rate.

The buoy can use a gyroscope or an accelerometer in order to estimate the underlying conditions (i.e., the wind surface speed) in real time. Also, depending on the positioning system used by the AUV and the operational conditions, the displacement and angular misalignment variances can be estimated. Lastly, given the estimated link parameters, the Tx beam divergence can be adjusted using a diffuser in front of the LED, whereas the Rx FoV can be changed using a mechanical iris in front of the Rx lens. For a practical implementation, the Tx beam-width and the Rx FoV could be selected amongst a discrete set of available values (using the closest values to the optimally calculated ones).

Future research concerns $\mathrm{Tx} / \mathrm{Rx}$ parameter optimization taking into account the effect of other channel impairments such as oceanic turbulence and solar background noise, as well as the dependence of the optical filter transmittance on the incidence angles of the optical rays.

\section{ACKNOWLEDGMENT}

This work was partly supported by Alex Ekwueme Federal University Ndufu-Alike (AE-FUNAI) in partnership with the Embassy of France in Nigeria and Campus France. The authors also acknowledge the support received from the French Embassy in Canada under the France Canada Research Funds program. The authors would like to thank Dr. Mohammad Taghi Dabiri from the Iran Telecommunication Research Center, Tehran, Iran, Mr. Oussama Haddad and Mr. Taha Essalih from Fresnel Institute, Marseille, France, and Mr. Abdallah S. Ghazy and Mr. Tau Raphael Rasethuntsa from McMaster University, Hamilton, Canada, for the fruitful discussions on channel modeling and analytical derivations, as well as Dr. Fabien Lemarchand from Fresnel Institute for discussions on the practical design of optical filters. They are also very grateful to Mr. Yves Chardard from SubseaTech Co., Marseille, and Mr. Christian Marfia from IFREMER, La Seyne-sur-Mer, France, for the practical considerations concerning practical AUV positioning parameters. This publication is also based upon work from COST Action CA19111 (European Network on Future Generation Optical Wireless Communication Technologies, NEWFOCUS), supported by COST (European Cooperation in Science and Technology). 


\section{REFERENCES}

[1] M. A. Khalighi, C. J. Gabriel, L. M. Pessoa, and B. Silva, Visible Light Communications: Theory and Applications. CRC-Press, 2017, ch. Underwater Visible Light Communications, Channel Modeling and System Design, pp. 337-372.

[2] N. Kaur, P. Singh, and P. Kaur, "Under water environment: A brief of explored work and future scope," IJCA International Conference on Advances in Emerging Technology, no. 2, pp. 12-17, Sept. 2016.

[3] H. Kaushal and G. Kaddoum, "Underwater optical wireless communication,” IEEE Access, vol. 4, pp. 1518-1547, Apr. 2016.

[4] S. Arnon, "Underwater optical wireless communication network," Optical Engineering, vol. 49, no. 1, pp. 1-6, Jan. 2010.

[5] G. Schirripa Spagnolo, L. Cozzella, and F. Leccese, "Underwater optical wireless communications: Overview," Sensors, vol. 20, no. 8, p. 2261, Apr. 2020.

[6] M. A. Khalighi, C. Gabriel, T. Hamza, S. Bourennane, P. Léon, and V. Rigaud, "Underwater wireless optical communication; Recent advances and remaining challenges," IEEE International Conference on Transparent Optical Networks (ICTON), pp. 1-4, July 2014, invited paper, Graz, Austria.

[7] F. Hanson and S. Radic, "High bandwidth underwater optical communication," Applied Optics, vol. 47, no. 2, pp. 277-283, Jan. 2008.

[8] B. M. Cochenour, L. J. Mullen, and A. E. Laux, "Characterization of the beam-spread function for underwater wireless optical communication links," IEEE Journal of Oceanic Engineering, vol. 33, no. 4, pp. 513521, Oct. 2008.

[9] B. Cochenour, L. Mullen, and J. Muth, "Temporal response of the underwater optical channel for high-bandwidth wireless laser communications," IEEE Journal of Oceanic Engineering, vol. 38, no. 4, pp. 730-742, Oct. 2013.

[10] M. Doniec, M. Angermann, and D. Rus, "An end-to-end signal strength model for underwater optical communications," IEEE Journal of Oceanic Engineering, vol. 38, no. 4, pp. 743-757, Oct. 2013.

[11] C. Gabriel, M. A. Khalighi, S. Bourennane, P. Léon, and V. Rigaud, "Monte-carlo-based channel characterization for underwater optical communication systems," IEEE/OSA Journal of Optical Communications and Networking, vol. 5, no. 1, pp. 1-12, Jan. 2013.

[12] T. Hamza, M. A. Khalighi, S. Bourennane, P. Léon, and J. Opderbecke, "Investigation of solar noise impact on the performance of underwater wireless optical communication links," Optics Express, vol. 24, no. 22 , pp. $25832-25845$, Oct. 2016.

[13] Y. Ata and Y. Baykal, "Structure functions for optical wave propagation in underwater medium," Waves in Random and Complex Media, vol. 24, no. 2, pp. 164-173, Feb. 2014

[14] Y. Baykal, "Scintillations of LED sources in oceanic turbulence," Applied Optics, vol. 55, no. 31, pp. 8860-8863, Nov. 2016.

[15] C. T. Geldard, J. Thompson, and W. O. Popoola, "A study of spatial and temporal dispersion in turbulent underwater optical wireless channel," IEEE ConTel Conference, pp. 1-5, July 2019, Graz, Austria.

[16] E. Zedini, H. M. Oubei, A. Kammoun, M. Hamdi, B. S. Ooi, and M.S. Alouini, "Unified statistical channel model for turbulence-induced fading in underwater wireless optical communication systems," IEEE Transcations on Communications, vol. 67, no. 4, pp. 2893-2907, Apr 2019.

[17] X. Sun, C. H. Kang, M. Kong, O. Alkhazragi, Y. Guo, M. Ouhssain, Y. Weng, B. H. Jones, T. K. Ng, and B. S. Ooi, "A review on practical considerations and solutions in underwater wireless optical communication," J. Lightw. Technol., vol. 38, no. 2, pp. 421-431, Jan. 2020.

[18] J. A. Simpson, B. L. Hughes, and J. F. Muth, "Smart transmitters and receivers for underwater free-space optical communication," IEEE Journal on Selected Areas in Communications, vol. 30, no. 5, pp. 964 974, June 2012.

[19] F. Mattoussi, M. A. Khalighi, and S. Bourennane, "Improving the performance of underwater wireless optical communication links by channel coding," Applied Optics, vol. 57, no. 9, pp. 2115-2120, Mar 2018.

[20] M. A. Khalighi, T. Hamza, S. Bourennane, P. Léon, and J. Opderbecke, "Underwater wireless optical communications using silicon photo-multipliers," IEEE Photonics Journal, vol. 9, no. 4, 2017, dOI 10.1109/JPHOT.2017.2726565.

[21] A. A. Farid and S. Hranilovic, "Outage capacity optimization for freespace optical links with pointing errors," J. Lightw. Technol., vol. 25, no. 7, pp. 1702-1710, 2007

[22] M. A. Khalighi and M. Uysal, "Survey on Free Space Optical Communication: A Communication Theory Perspective," IEEE Commun. Surveys \& Tutorials, vol. 16, no. 4, pp. 2231-2258, Nov. 2014
[23] C. Gabriel, M. A. Khalighi, S. Bourennane, P. Léon, and V. Rigaud, "Misalignment considerations on point-to-point underwater wireless optical links," IEEE OCEANS Conference, June 2013, Bergen, Norway.

[24] R. A. Khalil, M. I. Babar, N. Saeed, T. Jan, and H.-S. Cho, "Effect of link misalignment in the optical-internet of underwater things," MDPI Electronics, vol. 9, no. 4, p. 646, Apr. 2020.

[25] J. Liu, Y. Dong, and H. Zhang, "On received intensity for misaligned underwater wireless optical links," IEEE OCEANS Conference, Apr. 2016, Shanghai, China.

[26] S. Arnon and D. Kedar, "Non-line-of-sight underwater optical wireless communication network," J. Opt. Soc. Amer., vol. 26, no. 3, pp. 530-539, Mar. 2009.

[27] Y. Dong, S. Tang, and X. Zhang, "Effect of random sea surface on downlink underwater wireless optical communications," IEEE Communications Letters, vol. 17, no. 11, pp. 2164-2167, Nov. 2013

[28] H. Zhang, Y. Dong, and L. Hui, "On capacity of downlink underwater wireless optical MIMO systems with random sea surface," IEEE Communications Letters, vol. 19, no. 12, pp. 2166-2169, Dec 2015.

[29] C. Cox and W. Munk, "Measurement of the roughness of the sea surface from photographs of the sun's glitter," J. Opt. Soc. Amer., vol. 44, no. 11 pp. 838-850, Nov. 1954

[30] A. S. Ghazy, S. Hranilovic, and M. A. Khalighi, "Angular MIMO for underwater wireless optical communications: Channel modelling and capacity," IEEE Canadian Workshop on Information Theory (CWIT), pp. 1-6, June 2019, Hamilton, ON, Canada.

[31] _ _Angular MIMO for underwater wireless optical communications: Link modelling and tracking," IEEE Journal of Oceanic Engineering, under revision.

[32] I. E. Lee, Z. Ghassemlooy, W. P. Ng, and M. A. Khalighi, "Join optimization of a partially coherent gaussian beam for free-space optical communication over turbulent channels with pointing errors," Optics Letters, vol. 38, no. 3, pp. 350-352, Feb. 2013.

[33] V. V. Mai and H. Kim, "Beam size optimization and adaptation for high-altitude airborne free-space optical communication systems," IEEE Photon. J., vol. 11, no. 2, pp. 1-13, Apr. 2019.

[34] M. T. Dabiri, S. M. S. Sadough, and M. A. Khalighi, "Channel modeling and parameter optimization for hovering UAV-based free-space optical links," IEEE J. Sel. Areas Commun., vol. 36, no. 9, pp. 2104-2113, Sep. 2018.

[35] D. Wu, Z. Ghassemlooy, W.-D. Zhong, M.-A. Khalighi, H. L. Minh, C. Chen, S. Zvanovec, and A. C. Boucouvalas, "Effect of optimal Lambertian order for cellular indoor optical wireless communication and positioning systems," Opt. Engr., vol. 55, no. 6, pp. 1-8, 2016.

[36] P. Léon, F. Roland, L. Brignone, J. Opderbecke, J. Greer, M. A. Khalighi, T. Hamza, S. Bourennane, and M. Bigand, "A new underwater optical modem based on highly sensitive silicon photo-multipliers," IEEE OCEANS Conference, June 2017, Aberdeen, UK

[37] M. Khalighi, H. Akhouayri, and S. Hranilovic, "SiPM-based underwater wireless optical communication using pulse-amplitude modulation,' IEEE Journal of Oceanic Engineering, vol. 45, no. 4, pp. 1611-1621, Oct. 2020.

[38] Introduction to SiPM, Technical Note. ON Semiconductor $^{\circledR} \quad-$ SensL, 2011 (Rev. 6.0, Feb. 2017), available at https://www.sensl.com/downloads/ds/TN\%20\%20Intro\%20to\%20SPM\%20Tech.pdf.

[39] C. Gabriel, M. A. Khalighi, S. Bourennane, P. Léon, and V. Rigaud, "Investigation of suitable modulation techniques for underwater wireless optical communication," International Workshop on Optical Wireless Communications (IWOW), pp. 1-3, Oct. 2012, Pisa, Italy.

[40] J. M. Kahn and J. R. Barry, "Wireless infrared communications," Proceedings of the IEEE, vol. 85, no. 2, pp. 265-298, Feb 1997.

[41] J. R. Barry, J. M. Kahn, W. J. Krause, E. A. Lee, and D. G. Messerschmitt, "Simulation of multipath impulse response for indoor wireless optical channels," IEEE Journal on Selected Areas in Communications, vol. 11, no. 3, pp. 367-379, April 1993.

[42] A. Morel and L. Prieur, "Analysis of variations in ocean color," Limnology and Oceanography, vol. 22, no. 4, pp. 709-722, July 1977.

[43] J. W. Giles and I. N. Bankman, "Underwater optical communication systems. Part 2 : Basic design considerations," Military Communications Conference (MILCOM), vol. 3, pp. 1700-1705, Oct. 2005, Atlantic City, NJ, USA.

[44] E. Sarbazi, M. Safari, and H. Haas, "Statistical modeling of singlephoton avalanche diode receivers for optical wireless communications," IEEE Transactions on Communications, vol. 66, no. 9, pp. 4043-4058, Sept. 2018. 
[45] F. Xu, M. A. Khalighi, and S. Bourennane, "Impact of different noise sources on the performance of PIN- and APD-based FSO receivers," COST IC0802 Workshop, IEEE ConTEL Conference, pp. 211-218, June 2011, Graz, Austria.

[46] T. Hamza and M. Khalighi, "On limitations of using silicon photomultipliers for underwater wireless optical communications," 2nd West Asian Colloquium on Optical Wireless Communications (WACOWC), pp. 74-79, Apr. 2019, Tehran, Iran.

[47] F. Xu, A. Khalighi, P. Caussé, and S. Bourennane, "Channel coding and time-diversity for optical wireless links," Optics Express, vol. 17, no. 2, pp. 872-887, Jan. 2009

[48] M. T. Dabiri, S. M. S. Sadough, and M. A. Khalighi, "FSO channel estimation for OOK modulation with APD receiver over atmospheric turbulence and pointing errors," Opt. Commun., vol. 402, pp. 577-584, 2017.

[49] Inverse $Q$ function - MATLAB qfuncinv. The MathWorks, Inc., 2020 (Ver. 9.8.0.1396136 (R2020a)), available at https://www.mathworks.com/help/comm/ref/qfuncinv.html.

[50] T. Elfouhaily, B. Chapron, K. Katsaros, and D. Vandemark, "A unified directional spectrum for long and short wind-driven waves," J. Geophys. Res., vol. 102, no. C7, pp. 781-796, July 1997.

[51] M. D. Soltani, A. A. Purwita, Z. Zeng, H. Haas, and M. Safari, "Modeling the random orientation of mobile devices: Measurement, analysis and LiFi use case," IEEE Transactions on Communications, vol. 67, no. 3, pp. 2157-2172, March 2019.

[52] J. N. Monks, L. Yue, B. Yan, B. Aldred, A. Hurst, and Z. Wang, "A wide-angle shift-free metamaterial filter design for anti-laser striking application," Optics Communications, vol. 429, pp. 53-59, 2018

[53] A. A. Purwita, M. D. Soltani, M. Safari, and H. Haas, "Impact of terminal orientation on performance in LiFi systems," in IEEE Wireless Communications and Networking Conference (WCNC), 2018, pp. 1-6.

[54] Y. S. Eroğlu, Y. Yapıcı, and İ. Güvenç, "Impact of random receiver orientation on visible light communications channel," IEEE Transactions on Communications, vol. 67, no. 2, pp. 1313-1325, Feb 2019.

[55] LUXEON Rebel Color Line datasheet DS68 20171106. LUMILEDS, 2017, available at https://www.lumileds.com/uploads/265/DS68-pdf.
[56] C. Mobley, Light and Water: Radiative Transfer in Natural Waters. Academic Press, 1994

[57] S. Q. Duntley, "Light in the sea," J. Opt. Soc. Am., vol. 53, no. 2, pp. 214-233, Feb 1963. [Online]. Available: http://www.osapublishing.org/abstract.cfm?URI=josa-53-2-214

[58] T. Komine and M. Nakagawa, "Fundamental analysis for visible-light communication system using LED lights," IEEE Transactions on Consumer Electronics, vol. 50, no. 1, pp. 100-107, Feb 2004.

[59] B-Series Fast, Blue-Sensitive Silicon Photomultiplier Sensors datasheet. SensL, 2013 (Rev. 3.1, Nov. 2015), available at http://www.sensl.com/downloads/ds/DS-MicroBseries.pdf.

[60] T. Hamza, M. A. Khalighi, S. Bourennane, P. Léon, and J. Opderbecke, "On the suitability of employing silicon photomultipliers for underwater wireless optical communication links," IEEE/IET International Symposium on Communication Systems, Networks and Digital Signal Processing (CSNDSP), July 2016, Prague, Czech Republic.

[61] S. Huang, S. M. Patanwala, J. Kosman, R. K. Henderson, and M. Safari, "Optimal photon counting receiver for sub-dead-time signal transmission," Journal of Lightwave Technology, vol. 38, no. 18, pp. 5225-5235, Sept. 2020.

[62] T. Essalih, M. A. Khalighi, S. Hranilovic, and H. Akhouayri, "Optical OFDM for SiPM-based underwater optical wireless communication links," MDPI Sensors, Special Issue on Visible Light Communication, Networking, and Sensing, vol. 20, no. 6057, pp. 1-28, Oct. 2020, invited Paper.

[63] ITU-T Recommendation G.975.1, (02/2004).

[64] Numerical integration - MATLAB integral. The MathWorks, Inc., 2020 (Ver. 9.8.0.1396136(R2020a)), available at https://www.mathworks.com/help/matlab/ref/integral.html.

[65] Subsonus USBL/INS Datasheet. Advanced Navigation, 2019 (Ver. 3.0), available at https://www.advancednavigation.com/sites/default/files/product _documents/Subsonus_Datasheet_0.pdf.

[66] Subsonus TAG Transponder Datasheet. Advanced Navigation, 2019 (Ver. 3.0), available at https://www.advancednavigation.com/sites/default/files/product documents/Subsonus Tag Datasheet 0.pdf. 